\title{
Signal coupling and signal integrity in multi-strip Resistive Plate Chambers used for timing applications
}

\author{
Diego Gonzalez-Diaz ${ }^{\mathrm{a}, \mathrm{b}, *}$, Huangshan Chen ${ }^{\mathrm{b}}$, Yi Wang ${ }^{\mathrm{b}}$ \\ ${ }^{a}$ GSI Helmholtzcenter for Heavy Ion Research, Darmstadt, Germany \\ ${ }^{\mathrm{b}}$ Department of Engineering Physics, Tsinghua University, Beijing, China
}

\section{Abstract}

We have systematically studied the transmission of electrical signals along several 2-strip Resistive Plate Chambers (RPCs) in the frequency range $f=0.1-3.5 \mathrm{GHz}$. Such a range was chosen to fully cover the bandwidth associated to the very short rise-times of signals originated in RPCs used for sub-100ps timing applications. This work conveys experimental evidence of the dominant role of modal dispersion in counters built at the 1 meter scale, a fact that results in large cross-talk levels and strong signal shaping. It is shown that modal dispersion appears in RPCs due to the intrinsic unbalance between the capacitive and the inductive coupling $C_{m} / C_{o} \neq L_{m} / L_{o}$. A practical way to restore this symmetry has been introduced (hereafter 'electrostatic compensation'), allowing for a cross-talk suppression factor of around $\times 12$ and a rise-time reduction by $200 p s$. Under conditions $\checkmark$ of compensation the signal transmission is only limited by dielectric losses, yielding a length-dependent cutoff frequency of around $1 \mathrm{GHz}$ per 2 meter for typical float glass -based RPCs $\left(\left.\tan \delta\right|_{\text {glass }}=0.025 \pm 0.005\right)$.

It is further shown that 'electrostatic compensation' can be achieved for an arbitrary number of strips as long as the nature of the coupling is 'short-range', that is an almost exact assumption for typical strip-line RPCs. Evidence for deviations from the dominant 'TEM propagation mode has been observed, although they seem to have negligible influence in practical signal observables. This work extends the bandwidth of previous studies by a factor of almost $\times 20$.

Key words: RPC, Time-of-flight, Multi-strip RPC, Multi-hit capability, Cross-talk, Electrically-long counters, Simulations, inhomogeneous transmission lines

$P A C S: 29.40$, Cs

\section{Introduction}

Cross-talk and signal integrity are, a priori, critical aspects for the operation of electrically-long Resistive Plate Chambers (RPCs) with readout based on long multisegmented electrodes. The sometimes called multi-strip multi-gap RPCs (MMRPCs in short), pioneered by the $4 \pi$-experiment [1] at GSI fall, exemplarily, into this (by no means small) category. In order to streamline the forthcoming discussion some preliminary considerations are needed. We need first to introduce, as in [2,3], the electrical length $\Lambda_{e}$ of a Resistive Plate Chamber. This is done based on the 'cutoff frequency' of the current induced by a single-electron avalanche in the absence of Space-Charge effects and at typical working conditions:

$$
I(t)=e^{S t} \theta(t)
$$

\footnotetext{
* Corresponding author.

Email address: D.Gonzalez-Diaz@gsi.de (Diego Gonzalez-Diaz ).
}

$$
|\mathrm{ft}(I(t))|=\frac{1}{\sqrt{(2 \pi f)^{2}+S^{2}}}
$$

where $\theta(t)$ represents the Heavyside step function, 'ft' denotes the Fourier Transform, $\|$ refers to the modulus of the bracketed complex function, and $S=(\alpha-\eta) v_{\text {drift }}$ stands for the ionization rate in the active gas. The latter serves as a definition, in the present context, of $\alpha$ and $\eta$ (the multiplication and attachment coefficients, respectively) and $v_{\text {drift }}$ (the drift velocity of the electron swarm). Curiously, since no assumption is made on the sign of $S$, eq. 2 is obviously identical to the modulus of the response function of a low-pass RC-circuit with $S=-1 / R C$.

The characteristic 'cutoff frequency' $f_{c}$ is usually defined as the frequency needed for a drop by a factor of $1 / \sqrt{2}$ (i.e., $-3 \mathrm{~dB}$ ) with respect to the $\mathrm{DC} /$ zero-frequency limited in the Fourier amplitude spectra, also often equivalently as the frequency needed for a factor $1 / 2$ drop in the Fourier power spectra, yielding thus $f_{c}=S / 2 \pi$ from eq. 2. Assuming, with sufficient generality for present purposes, that signal 
propagation takes place at approximately half the speed of light, the electrical length of an RPC of length $D$ can be estimated as:

$\Lambda_{e}=\frac{D}{\lambda_{c}}=\frac{f_{c}}{v} D \simeq \frac{S}{\pi c} D$

where $c$ is the speed of light and $D$ is the counter length.

With approximate character, an electrically-long structure is customary defined as that fulfilling the condition $\Lambda_{e}>1$ [3]. It is indeed possible to quantitatively understand the implication of the above fact thanks to the recently measured parameters of the swarm [4] for the main $\mathrm{RPC}$ gas component $\left(\mathrm{C}_{2} \mathrm{H}_{2} \mathrm{~F}_{4}\right)$. These measurements were performed at low-pressure and are thus largely free of saturation effects, corresponding approximately to the situation sketched in eq. 1 . When extrapolated to typical working conditions $\left(T=20^{\circ} \mathrm{C}, P=1 \mathrm{~atm}\right)$, the parameters measured in [4] provide the following lower bounds for the length $D^{*}$ above which an RPC can be considered to be electrically-long:

$$
\begin{aligned}
& D_{\text {trigger }}^{*}(E=50 \mathrm{kV} / \mathrm{cm})=\frac{\pi c}{S(E=50 \mathrm{kV} / \mathrm{cm})}=80 \mathrm{~cm} \\
& D_{\text {timing }}^{*}(E=100 \mathrm{kV} / \mathrm{cm})=\frac{\pi c}{S(E=100 \mathrm{kV} / \mathrm{cm})}=5 \mathrm{~cm}
\end{aligned}
$$

Eqs. 4, 5 have been evaluated for two typical field values corresponding to RPCs used for trigger, $E \simeq 50 \mathrm{kV} / \mathrm{cm}$ [5], and timing, $E \simeq 100 \mathrm{kV} / \mathrm{cm}[6]$. In particular, the cutoff frequency obtained for timing RPCs is as high as $f_{c} \simeq 3 \mathrm{GHz}$. The signal bandwidth is thus reasonably met by the fast front end electronics used in the so far existing strip timing RPC walls from the $4 \pi$-experiment[7] $(\mathrm{BW} \simeq 1.5 \mathrm{GHz})$ and $\mathrm{HADES}[8](\mathrm{BW} \simeq 2 \mathrm{GHz})$. More realistically, rise-time measurements performed directly over RPC signals with a 2-stage $\sim 2 \mathrm{GHz}$-bandwidth amplifier at typical pressure, gas mixture and $\mathrm{HV}$, as in [9], have yielded a value of $f_{c}=$ $1.4 \mathrm{GHz}$ at $100 \mathrm{kV} / \mathrm{cm}$. The $1 / 2$ lower signal bandwidth as compared to the one from [4] may be interpreted as a contribution of both the presence of Space-Charge for typical working thresholds and the limited bandwidth of the readout system, as has been argued by authors [10]. ${ }^{1}$

It is thus expected, on very general grounds, that extreme conditions for signal transmission take place, most prominently, in the $0.9 \mathrm{~m}$-long multi-strip wall of the $4 \pi$ experiment [1] $\left(\Lambda_{e}=18\right)$, the 1.6m-long 2-strip counter developed by Fonte in 2002 [11] and, more recently, in the 1.6m-long counters of the EEE project [12](both $\left.\Lambda_{e}=32\right) .{ }^{2}$ Second generation multi-strip counters like CBM-ToF [13], R3B-neuLAND [14], R3B-iTOF [15] or

\footnotetext{
1 The estimate of $f_{c}$ from the signal rise-time is not affected by a mere bandwidth reduction of the measuring device as long as the signal is purely exponential. This has been shown in [9] for lineal devices. Deviations from the exponential growth at the discrimination point are thus needed in order to reduce $f_{c}$.

2 The HADES wall is not included in this list due to its singlestrip design, that makes signal transmission a far simpler problem. HADES longest strip is $\Lambda_{e} \simeq 11$-long.
}

the STAR-MTD upgrade [16] are presently planned to be built based on multi-strip designs with lengths in the range $0.5-2 \mathrm{~m}\left(\Lambda_{e}=10-40\right)$. Although single-strip designs like the HADES time-of-flight (ToF) wall offer a safe alternative from the point of view of signal transmission, being virtually cross-talk free [17], it is at present questionable whether such an approach is practical for large numbers of cells, in view of the 'manpower $\times$ time' overhead.

Of the above experiments, intrinsically multi-hit environments like CBM (3-5\% occupancy per strip per event, $\simeq$ 1000 tracks per event), speak against large cluster sizes that may typically arise either during signal induction or due to sustained capacitive and inductive coupling during signal transmission over many electrical lengths, i.e., cross-talk. Efficiency measurements on the 2-strip, 1.6m-long prototype of [11] point, indeed, to very high cross-talk levels (80-90\%) while the $4 \pi$-experiment has recently reported a cluster size of the order of 4.5 strips/track [18]. There is additional evidence that the cluster sizes measured in the earlier case are generated indeed during signal transmission [2] while the ones measured in the latter have certainly a sizable contribution from the avalanche footprint (see next section). Remarkably, it has been shown by the EEE collaboration that reducing the system bandwidth from $f_{c} \simeq$ $3 \mathrm{GHz}$ to $f_{c} \simeq 300 \mathrm{MHz}$ (amplifier peaking time $\simeq 1 \mathrm{~ns}$ ), i.e., a $\sim 1 / 10$ bandwidth reduction, is compatible with preserving a good time resolution and efficiency for long strips, thus largely relaxing condition (5) by approximately the same factor and easing transmission. ${ }^{3}$ Yet, the low filling factor (strip to pitch ratio) of less than $80 \%$ used in those counters [12] (presumably stemming from cross-talk optimization) together with the observed position-dependent space-resolution along the strips do not ensure the uniformity of response; the absence of experimental information on the shear cross-talk levels and cluster sizes do not allow to take a good multi-hit performance for granted, either. Thus, none of these scenarios seem to be technologically satisfactory for a high multiplicity experiment like, for instance, CBM.

Besides the aforementioned developments, systematic studies on signal propagation in RPCs are very limited. Numerical simulations have been performed for the Pestov counter [19] while systematic measurements are available for trigger-type RPCs [20], where the main phenomena ruling signal coupling in multi-strip counters have been unambiguously identified. The work of W. Riegler in 2002 [21], combining experimental observations and simulations, remains possibly the most complete up to date, despite covering a modest $200 \mathrm{MHz}$ bandwidth and being performed for trigger-type RPCs. Not only cross-talk but specially dielectric losses (due to the presence of float glass) remain thus to be assessed in timing-type RPCs up to $f_{c} \simeq 3 \mathrm{GHz}$.

This work is structured as follows: in section 2 we discuss cluster sizes in multi-strip RPCs, identifying the contribu-

\footnotetext{
3 As recalled in an earlier footnote, this statement is dependent, in detail, on the signal shape.
} 
tions from the avalanche induction profile (charge sharing) and from the signal transmission (cross-talk). In section 3 we present systematic pulser/scope-based measurements of transmission properties for 2-strip RPCs; based on the structure of the solutions for the loss-less transmission line problem [21], a novel scheme for cross-talk compensation is introduced. In sections 4 and 5 we discuss the generalization of this concept to lossy N-strip systems. For that, in section 4 we identify the dielectric losses by measuring the RPC frequency response with a large bandwidth network analyzer, giving a simple theoretical prescription on how to include them into the loss-less solutions. Following, in section 5, an extension of the aforementioned compensating scheme is given for N-strips, with particular focus on the literal solutions for 5 -strip structures. A discussion on the results and the conclusions of the work are given in sections 6 and 7 .

\section{Cluster sizes in multi-strip RPCs}

Large cluster sizes either restrict the track identification capability of a time-of-flight detector or proportionally increase its cost. This is so because conceptual designs of this type of detectors, unlike tracking detectors, are usually based on the average system occupancy as their main figure of merit. The latter is nothing else but the average probability that a detection cell/strip is hit per event, thus the ratio of the average number of tracks per event to the number of active cells $\bar{n}=N_{\text {tracks }} / N_{\text {cells }}$. For a Poissondistributed track multiplicity, the fraction of cells per event over which more than one track will imping can be calculated simply as:

$P_{>1}=1-P_{0}-P_{1}=1-(1+\bar{n}) e^{-\bar{n}}$

assuming that each track produces a measurable signal in only one cell. The probability of finding a cell with more than one hit $\left(P_{>1}\right)$ amongst all the fired cells is usually referred to as the 'double hit probability':

$P_{\text {double }}=\frac{P_{>1}}{P_{1}+P_{>1}}=\frac{1-(1+\bar{n}) e^{-\bar{n}}}{1-e^{-\bar{n}}} \simeq \bar{n}$

where the assumption $\bar{n} \ll 1$ has been made to derive the right-hand-side identity. Very often it is not possible to identify any of the two tracks arriving at the same cell so $2 P_{\text {double }}$ represents, approximately, the contamination from wrongly identified tracks that unavoidably goes into the physics analysis (or the track-matching inefficiency, in case tracks hitting those cells are discarded). Once an acceptable value for $P_{\text {double }}$ is fixed by the physics goals (for instance, $5 \%$ ), preliminary cost estimates can be performed (in approximate linear proportionally with the number of required cells).

In the more realistic case where each track causes in average a certain number of strips to fire $\left(\bar{n}_{s}\right),{ }^{4}$ eq. 7 must be replaced by:

\footnotetext{
4 This is, incidentally, the definition of cluster size.
}

$P_{\text {double }}=\frac{1-\left(1+\frac{N_{\text {tracks }}}{N_{\text {cells }}} \bar{n}_{s}\right) e^{-\frac{N_{\text {tracks }}}{N_{\text {cells }}} \bar{n}_{s}}}{1-e^{-\frac{N_{\text {tracks }}}{N_{\text {cells }}} \bar{n}_{s}}}$

The number of cells required for keeping $P_{\text {double }}$ close to a certain design value in an environment with a given number of tracks increases, thus, in direct proportionality with the cluster size, unless an increase of $P_{\text {double }}$ as a function of $\bar{n}_{s}$, according to eq. 8 , is accepted. Besides the shear occupancy problem, a track crossing a timing RPC may affect more than just $\bar{n}_{s}$ strips. The electromagnetic perturbation on the strips potential, even if below threshold, can affect the time measured for a coincident track in an uncontrolled way. This possibility has been studied so far, perhaps strikingly, only for single-strip structures [17], [28] where the effect should be absent. Even in these almost ideal conditions, a slight degradation of the time resolution could still be seen in [17] affecting the first neighbors' performance under multi-hit conditions, despite the cross-talk levels were as small as $0.4 \%$.

A priori, the most obvious candidate for increasing the RPC cluster sizes is the spatial spread of the avalanche charge. Although no experimental value for the transverse diffusion coefficient $D_{T}$ exists for the standard RPC gas mixture, an estimate can be made based on the one recently measured for the longitudinal diffusion coefficient $D_{L}$ in pure $\mathrm{C}_{2} \mathrm{H}_{2} \mathrm{~F}_{4}$ [4] by using the ansatz $D_{T} \simeq D_{L}$, that is a good approximation at high fields. This yields a typical avalanche diffusion radius $r=\sqrt{D_{T} g / v_{d}} \simeq 10 \mu \mathrm{m}$ for a gap $g=0.3 \mathrm{~mm}$ under $E=100 \mathrm{kV} / \mathrm{cm}$ (and $r \simeq 30 \mu \mathrm{m}$ for $g=2 \mathrm{~mm}, E=50 \mathrm{kV} / \mathrm{cm})$. Given the typical scale of the read-out strips $(\sim \mathrm{cm})$, the avalanche diffusion can be thus expected to have a very minor role, indeed, in the observed cluster sizes.

Experimentally first [20] and latter theoretically [21],[22], [23], [24], the two main electrostatic effects that can dominate the cluster sizes and the signal shapes in multi-strip RPCs have been identified. Following [24], in the next subsection we discuss, semi-quantitatively, cluster sizes originated from the induction process.

\subsection{Charge-sharing}

In the most general case nowadays, where the high voltage (HV) is applied through a low-conductive coating of surface resistivity $R_{s}$ (for instance, [25]), four paradigmatic situations can occur from the point of view of signal induction (Fig. 1):

(i) The characteristic avalanche duration time $\tau_{a v} \simeq g / v_{d}$ is much larger than the response time of the HV coating $\tau_{H V}$ under which the latter behaves in practice like a perfect conductor $\left(\tau_{a v} \simeq 1.5 \mathrm{~ns}\right.$ for $g=0.3 \mathrm{~mm}$, $E=100 \mathrm{kV} ; \tau_{a v} \simeq 18 \mathrm{~ns}$ for $g=2 \mathrm{~mm}, E=50 \mathrm{kV}$, as from [4]). The induction can be seen as taking place on the HV electrode (see left dotted current generator in Fig. 1 up-left) that is capacitively coupled to the read-out strips in a high-pass configuration. When 
the coupling between $\mathrm{HV}$ and readout strips is ideal $\left(C_{\text {ins }}=\infty\right)$ all the strips see the same signal, proportional to the counter capacitance per unit area $C_{A}$ divided by the number of strips.

(ii) The characteristic avalanche duration time $\tau_{a v}$ is much smaller than $\tau_{H V}$. The avalanche induces currents in the electrodes according to the 'weighting fields' $E_{w}$ obtained upon application of the Ramo theorem [26]. The weighting fields determined in that way can still, in specific geometries, cause sizeable cluster sizes.

(iii) For completeness, an abstraction can be made on case i), by imposing the additional condition that the coating represents an ideal ground (a situation not obviously realizable in practice). In this case, the signal disappears at the coating, by definition, thus screening the readout strips completely.

(iv) At last we can consider the avalanche duration to be just larger than $\tau_{H V}$ (intermediate situation). A timedependent weighting field must be then calculated. A simple prescription is given in [24] on how to do this, together with practical examples for 1-gap chambers. As simple as it may be, no attempt has been made, so far, to verify this model.

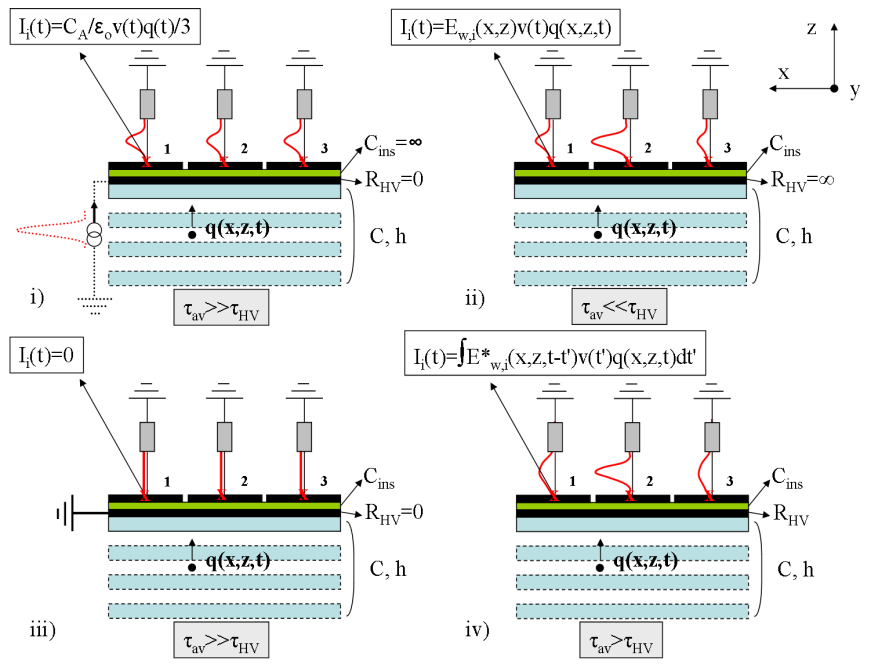

Fig. 1. The four cases discussed in this section regarding signal induction: i) The HV coating behaves like an ideal conductor, yielding equal charge sharing among all strips, ii) the HV coating behaves like an ideal insulator, and the induction profile can be obtained from a standard static weighting field calculation (units $\left[L^{-1}\right]$ ), iii) the read-out strips are fully shielded, iv) the presence of the HV coating has to be included for evaluation of the induction process yielding time-dependent weighting fields (units $\left[L^{-1} T^{-1}\right]$ ).

It is important to realize an implicit assumption made in [24], and thus in cases i)-iv): the region affected by the induction process $r_{\text {ind }}$ must be electrically short $r_{\text {ind }} \leq 5 \mathrm{~cm}$ (from eq. 5). Otherwise, field propagation must be obviously taken into account. This fact highlights the difficulty of addressing cases i) and iii) in a practical situation. ${ }^{5}$ For

\footnotetext{
5 For instance, the theorems obtained in [24] predict an equal signal sharing for infinite strips placed over an infinite floating conductor.
}

the geometries discussed here, however, and for most practical cases, it is reasonable to assume that $r_{i n d} \leq 5 \mathrm{~cm}$ (see for instance the weighting field profiles obtained in two extreme scenarios in Fig. 2).

Under the, usually desired, condition $\tau_{a v} \ll \tau_{H V}$, the cluster size originated during induction is minimal and can be obtained from a static weighting field profile determined with the Ramo theorem alone (case ii)). This situation is often given in practice. In order to better understand the implications of this condition, we take the expresion for $\tau_{H V}$ obtained in [24]:

$\tau_{H V} \simeq R_{s} \epsilon_{0} h$

where $h$ can be interpreted as the anode cathode-distance and $R_{s}$ is the surface resistivity of the material in $[\Omega / \square]{ }^{6}$ We take, for illustration, a typical value of $h=4 \mathrm{~mm}$, that corresponds to a $2 \mathrm{~mm}$-bakelite $/ 2 \mathrm{~mm}$-gap trigger RPC and an $0.5 \mathrm{~mm}$-glass $/ 0.3 \mathrm{~mm}$-gap 6-gap timing RPC. Thus, the condition $\tau_{a v} \ll \tau_{H V}$ requires values for the resistivity of the coating $R_{s}>500 \mathrm{k} \Omega / \square$ and $R_{s}>50 \mathrm{k} \Omega / \square$ for trigger and timing RPCs, respectively. Coatings at the scale of 10 $\mathrm{M} \Omega / \square$ (for instance [13]) seem to be thus very fitting.

For illustration, weighting fields for two distinct RPCs are calculated as in [2]. ${ }^{7}$ These geometries are characterized by two extreme values of the ratio of strip width to anode-cathode distance: $\mathrm{w} / h \simeq 0.3 \ll 1[1]$ and $\mathrm{w} / h \simeq$ $5 \gg 1[6]$, generating thus very different profiles across the strip, as shown in Fig. 2.
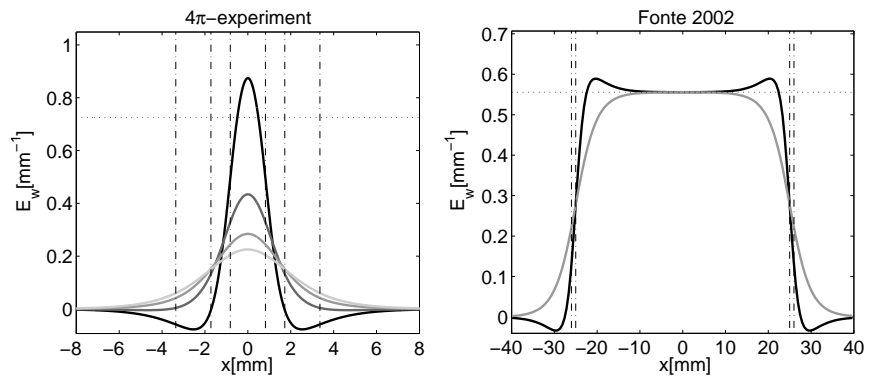

Fig. 2. Weighting field profiles across the strips ( $x$ coordinate), as obtained for a strip centered at 0 , evaluated at the center of each gap, for the $4 \pi$-experiment (left) and Fonte-2002 prototype (right). Lines shown in gray gradient from black (gap closest to the read-out strip) to light gray (gap furthest from the read-out strip). The dashed line shows $E_{w}=C_{A} / \epsilon_{0}$.

\footnotetext{
However, any signal registered on the strips placed at infinite can only arrive after an infinite time, since the induction can not take place faster than the speed of light.

6 This expression is exact only for 1-gap chambers without resistive plates, for which the problem could be analytically solved in [24]. Since i) the time-dependent component of the weighting field is exponentially suppressed with $\tau_{H V}$ [24], ii) the dielectric constant of the resistive plates is at most a factor $\times 10$ higher than the one of the gas gap, and iii) the present discussion is based on orders of magnitude, the approximate expression for $\tau_{H V}$ in eq. 9 is kept.

7 In these two detectors the signals are directly read-out from the electrodes on HV-potential. The HV plane does not cause therefore signal screening, and the situation is analogous to case ii) in Fig. 1.
} 
In order to determine the region over which a strip can effectively 'see' an avalanche signal (i.e., the signal being above threshold) a full simulation is needed [2]. This approach is, however, outside the scope of the present work. An approximate idea of the size of the induction phenomena can be obtained by calculating the region $\Delta X$ over which the average weighting field drops to a large fraction of its maximum value ( $10 \%$, for instance). The area of influence of a strip beyond its geometrical limits can be estimated after substracting its width: $r_{i n d}=\Delta X-\mathrm{w}$. This yields $r_{\text {ind }} \simeq 1$.4pitch for [1] and $r_{i n d} \simeq 0.1$ pitch for [6]. Reciprocally, 'how far a strip can see' is directly related to 'from how far an avalanche can be seen', thus to the cluster size. Intuitively, considerations on the resistance of the HV layer apart, the condition $\mathrm{w} / h \gg 1$ is thus expected to minimize the cluster size originated during induction. A detector not fulfilling $\mathrm{w} / h \gg 1$ may additionally increase its cluster size by mere geometrical considerations. E.g., assuming an angle of incidence in the plane transverse to the strips of $\theta=$ $30 \mathrm{deg}$ with respect to perpendicular incidence, the track projection over the strips plane would be $r_{i n d, \theta} \simeq 2 h \tan \theta$ yielding $r_{i n d, \theta}=2.3$ pitch for [1] and $r_{i n d, \theta}=0.22$ pitch for $[6]$.

Although negative weighting fields can yield induced signals of opposite polarity, specially for avalanches in the region between the strips and close to them (Fig. 2), the net signals originated during induction have generally the same polarity, thus constituting effectively an area over which the avalanche-induced charge is 'spread'. We will refer to this phenomena as 'charge sharing' (or 'avalanche foot-print'), to make clear the different underlying principle with respect to the main phenomena of interest in this work, that is introduced in the next section.

\subsection{Cross-talk}

According to [24] the finally measured currents and voltages can be determined once the currents induced at the electrodes have been calculated (as in Fig. 1) but only after introducing all the resistive, capacitive and inductive elements present in the system, including the RPC itself. The associated circuit problem must be then solved using as input the calculated currents modeled as ideal generators. In case of being electrically-long, however, an RPC can not be characterized by conventional circuit theory and a distributed circuit theory is needed, where electrostatic elements per unit length are used as input parameters (see Fig. 3 for a simple 2-strip situation). The argument sketched above suggests a separation between the transverse and longitudinal signal dynamics and will be followed here for the sake of simplicity, as in [2]. Going beyond this assumption requires of a 3 -dimensional modeling of the structure.

We discuss first the simpler case of signal transmission in loss-less structures (only capacitive and inductive elements are present). As shown in the next section, the main transmission patterns are indeed emerging from the structure of the solutions to this problem. A dedicated discussion on dielectric losses (due to the shunting conductances $G_{g}, G_{m}$ in Fig. 3) is postponed to section 4 . The presence of skin effect would yield additional resistive elements in Fig. 3 but it is shown (also in section 4) to be a minor effect and has been neglected for the sake of providing a simpler image of the process.

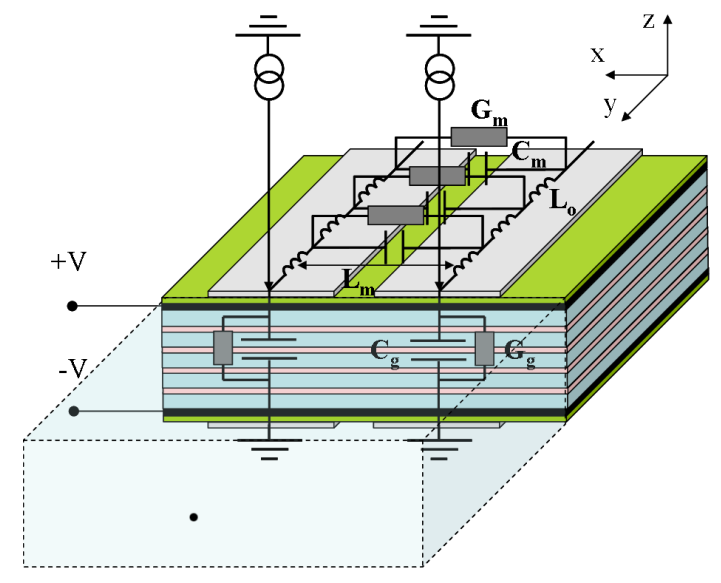

Fig. 3. Illustration of the transmission problem in 2-strip RPCs. The currents may be obtained with the formalism of section 2.1 , for instance.

The analytical solutions of the N-strip transmission line equations (often named 'the telegrapher's equations') for the loss-less case can be found, for instance, in the excellent overview book of C. R. Paul [3]. They were introduced in the Resistive Plate Chamber field by W. Riegler in 2002 [21] in the context of impedance-matched systems. A straightforward generalization of the formalism in [21] in order to explicitly take into account the reflections was given in [2] for the 2-strip case. For the N-strip case the complete solution can be compactly written as: ${ }^{8}$

$$
\vec{I}_{T}(t)=\frac{\hat{Z}_{i n}}{R} \frac{\hat{T}}{2} \sum_{j=0}^{\infty}(1-\hat{T})^{j} \hat{M}\left(\begin{array}{c}
\hat{M}_{1 n}^{-1} I\left(t-\frac{(-1)^{j} y_{0}+2\lceil j / 2\rceil D}{v_{1}}\right) \\
\ldots \\
\hat{M}_{N n}^{-1} I\left(t-\frac{(-1)^{j} y_{0}+2\lceil j / 2\rceil D}{v_{N}}\right)
\end{array}\right)
$$

where $\vec{I}_{T}(t)$ is the $N$-dimensional array of currents measured through the corresponding resistances $R$ placed at $y=0$ when the N-strip structure is excited along line $n$ by a current $I(t)$ originated at position $y=y_{0} \cdot{ }^{9}$ The sum extends over all $j$ reflections and $\lceil j / 2\rceil$ denotes the next higher integer of $j / 2 . \hat{T}$ is the (in-out) transmission coefficient of the line:

$\hat{T}=2 \hat{Z}_{c}\left(\hat{Z}_{i n}+\hat{Z}_{c}\right)^{-1}$

$\hat{Z}_{c}$ is the characteristic impedance matrix of the transmission line and $\hat{Z}_{i n}$ is the impedance matrix with which it is

\footnotetext{
8 In the following, a variable with hat ${ }^{\wedge}$ denotes an $N \times N$ matrix.

9 We assume that all ports are read-out with the same electronic system, having an input resistance $\mathrm{R}$. In the most general case where several read-out systems are present $R$ should be replaced by a vector.
} 
loaded, that must be calculated according to [21]. In the most typical case where all ports are terminated with the amplifier input resistance $R$ :

$\hat{Z}_{\text {in }}=\hat{1} R$

and the pre-factor in eq. 10 becomes $1 . \hat{M}$ is the matrix of eigenvectors with elements $\hat{M}_{i j}$ and $\vec{v}=\left\{v_{1}, \ldots, v_{N}\right\}$ the array of the inverse squares of the eigenvalues of the following diagonalization problem:

$\hat{M}^{-1}(\hat{C} \hat{L}) \hat{M}=(\hat{1} \vec{v})^{-2}$

with the impedance matrix being defined as:

$\hat{Z}_{c}=\hat{L} \hat{M}(\hat{1} \vec{v}) \hat{M}^{-1}$

The notation $\hat{M}_{i j}^{-1}$ indicates the element with column/row indexes $i / j$ of the inverse matrix $\hat{M}^{-1} . \hat{L}$ and $\hat{C}$ are the 'per unit-length' inductance and capacitance matrices of the structure and $\hat{1}$ is the unit-matrix. Under the (most usual) situation where all materials have a relative magnetic permeability $\mu_{r} \simeq 1$, the following relation applies [27]:

$\hat{L}=\frac{1}{c^{2}} \hat{C}_{0}^{-1}$

Here $\hat{C}_{0}$ is the capacitance matrix of the transmission line with all dielectrics replaced by empty space, an equivalence that will be used throughout this document. A description of the structure of the matrices $\hat{C}$ can be found in section 5 or in [3]. It must be noted that for parallel-plate transmission lines, even in case of a homogeneous surrounding medium, analytical calculations of the elements of the capacitance matrices exist in just few cases. For a parallelplate structure that is also inhomogeneous, a numerical solution is enforced.

There are some relevant properties that emerge only after including the reflections explicitly (as in eq. 10), and the most evident ones have been discussed in [2], among them the 'delayed cross-talk' (the fact that cross-talk stemming from a reflection at the opposite strip-end can largely exceed the direct cross-talk) and the charge conservation (meaning that the charge induced in the main strip is collected after summing up all the reflections and, under the same conditions, cross-talk does not transport net charge between the strips either), a statement that is proved in the appendix of this work. This fundamental property of cross-talk in loss-less lines (the absence of net charge when integrating over a large time window) has been used in [11] in order to experimentally demonstrate its importance in multi-strip RPCs.

Eq. 10, in spite of being analytical, is not very useful in its present form. The long and tedious algebraic computation can be carried out in a symbolic way, with Mathematica [36] for instance, but it is very difficult to grasp the meaning of the multiple terms arising and how an optimization can be realized in practice. Solutions are often just too general, while the particular application my fall easily under a set of reasonable simplifying conditions, making the solutions of eq. 10 more enlightening as well as the fundamental variables ruling the phenomena. Following [3], we will call this type of solutions 'literal' solutions. Literal solutions for an inhomogeneous un-matched 2-strip line can be found, for instance, under the low-coupling approximation [37], [2] and their properties are discussed in the next section.

\section{A systematic study of cross-talk and signal integrity in 2-strip counters}

\subsection{Introduction to the problem}

It has been recently reported [2] that modal dispersion could be responsible for the extreme cross-talk patterns observed in early implementations of timing Resistive Plate Chambers with multi-strip readout [11]. Modal dispersion emerges from the structure of the solutions of N-conductor lossless transmission lines (eq. 10) when transmission is performed through inhomogeneous dielectric structures. As a matter of fact, an RPC is intrinsically an inhomogeneous transmission line. This very relevant feature can not be altered in view of the simultaneous need of amplifying gas $\left(\epsilon_{r}=1\right)$ and HV insulator (either float glass or Bakelite, $\left.\epsilon_{r}=5-10\right)$.

In the following we will assume that the reader is familiar with the literal solutions to the exemplary 2 -strip problem in the form presented in [2]. They represent a particular case of the general N-strip situation in eq. 10, where the diagonalization problem is particularly easy. Following [2], the subsequent discussion can be stream-lined by recalling the '2-strip parameters':

(i) Propagation velocity $\bar{v}$ (average velocity of the two system modes).

(ii) Velocity dispersion $\Delta v / \bar{v}$ (relative velocity difference of the two system modes).

(iii) Characteristic impedance $Z_{c}$ (diagonal element of the characteristic impedance matrix).

(iv) Coupling coefficient $Z_{m} / Z_{c}$ (ratio of the non-diagonal element of the impedance matrix to the diagonal one). A deeper insight can be obtained by recalling their approximate expressions under the condition $Z_{m} / Z_{c}<1$ (low coupling):

$$
\begin{aligned}
& \bar{v} \simeq \sqrt{\frac{C_{g 0}+C_{m 0}}{C_{g}+C_{m}}} c, \quad \frac{\Delta v}{\bar{v}} \simeq \frac{C_{m}}{C_{g}+C_{m}}-\frac{C_{m 0}}{C_{g 0}+C_{m 0}} \\
& Z_{c} \simeq \frac{1}{\sqrt{\left(C_{g 0}+C_{m 0}\right)\left(C_{g}+C_{m}\right)}} \frac{1}{c} \\
& \frac{Z_{m}}{Z_{c}} \simeq \frac{1}{2}\left(\frac{C_{m}}{C_{g}+C_{m}}+\frac{C_{m 0}}{C_{g 0}+C_{m 0}}\right)
\end{aligned}
$$

They depend on the capacitance with respect to ground $C_{g}$ and the mutual capacitance $C_{m}$ per unit length both in the real structure and in the empty space $\left(C_{g 0}, C_{m 0}\right)$ and can be obtained by solving the corresponding 2-dimensional electrostatic problems for a cross-section of the device (Fig. 4- 
middle). In the limit $C_{m}, C_{m 0} \rightarrow 0$ the well-known 1-strip (1 conductor +1 reference) expressions for $\bar{v}$ and $Z_{c}$ are recovered by recalling that the induction coefficient per unit length is then $L_{0}=1 /\left(c^{2} C_{g 0}\right)$. Signal transmission and cross-talk in a 2 -strip line (2 conductors +1 reference) can be expressed conveniently as a function of the parameters above, as shown in [2]. Other descriptions of the loss-less 2-strip situation are however possible: as an example, classical circuit models based on odd and even impedances and velocities exist since long time [34], while more tractable literal solutions for a general un-matched case (similar to [2]) can be found more recently ([37], for instance). An approximate, although insightful, derivation can be found in [35] and references therein.

In an inhomogeneous structure the velocity/modal dispersion given by eq. 16-right can dominate the cross-talk and transmission patterns well beyond the shear strength of the electrostatic coupling $Z_{m} / Z_{c}$ (eq. 18). Indeed, its importance depends critically on the propagation distance and the signal rise-time. As it can be deduced from eq. 16, the velocity dispersion is zero for a homogeneous material with arbitrary dielectric constant $\epsilon=\epsilon_{0} \epsilon_{r}$, since $C_{g}=$ $\epsilon_{r} C_{g 0}, C_{m}=\epsilon_{r} C_{m 0}$. Not being this statement generally true for an inhomogeneous structure, the value of $\Delta v / \bar{v}$ may be, however, 'adjusted'. A simple implementation of this idea is shown in Fig. 4-up: values in empty space are not changing due to the presence of an additional dielectric above the readout electrodes, the coupling to ground is virtually unaffected, and only $C_{m}$ varies. The labels refer to 3 paradigmatic cases: 'under-compensated' $(\Delta v / \bar{v}<$ $0)$, 'compensated' $(\Delta v / \bar{v}=0)$ and 'over-compensated' $(\Delta v / \bar{v}>0)$. A system is thus said to be compensated when the coupling coefficient $Z_{m} / Z_{c}$ is the same in the filled and in the empty structure, $Z_{m} / Z_{c}=Z_{m} /\left.Z_{c}\right|_{0}$, as can be deduced from eqs. 16 and 18. The velocity dispersion of the two system modes is then zero and, equivalently, the capacitive and inductive coupling are balanced $C_{m} /\left(C_{g}+C_{m}\right)=$ $L_{m} / L_{0}$, through relation 15 . This symmetry was realized long ago, but it is usually regarded as a feature proper only of homogeneous lines [35]. It is therefore very appealing to explore the possibility of constructing an RPC fulfilling the condition $\Delta v / \bar{v}=0$. Such a compensated system theoretically exhibits minimal signal shaping and cross-talk, being its properties independent from the propagation distance, as long as losses can be neglected.

Several caveats to the above interpretation are worth being noted at this point. First, transmission through inhomogeneous structures precludes a pure TEM-mode propagation (where the electric and magnetic fields are transverse to the direction of movement) and thus formally invalidates the telegrapher's equations and the solutions given in eq. 10. Moreover, the loss-less assumption that, if violated, also invalidates eq. 10, was not proved for neither typical glass nor Bakelite -based RPCs. It is a common practice to include these aforementioned facts under the name 'quasi-TEM' approach and to use the telegrapher's equations anyway, however a sound experimental measure-

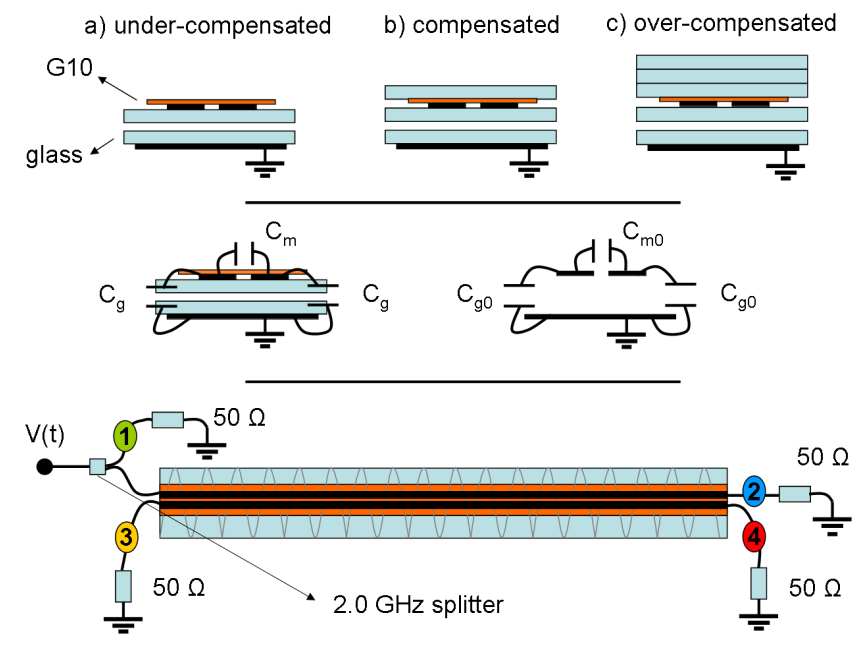

Fig. 4. Up: Transverse section of the three typical cases studied in this work, from left to right: a system with negative velocity dispersion (under-compensated), zero (compensated) and positive (overcompensated). Middle: description of how to calculate the electrostatic parameters needed for solving the loss-less transmission line problem. Down: Up view of the scheme used for the measurements presented in this section. A fast pulser signal $V(t)$ (output resistance $50 \Omega$ ) is connected to the left of the structure via a $2 \mathrm{GHz}$-splitter (port 1). All 4 ports are measured simultaneously with a $2.5 \mathrm{GHz}$

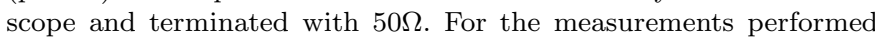
with a $3.5 \mathrm{GHz} 4$-port network analyzer, the connections were made directly to the structure, without additional elements.

ment is then required. Eq. 10 is also invalidated in the presence of frequency or direction-dependent electrical properties, that is sometimes the case in dielectric materials. Last but not least, sample-to-sample variations of the electrical properties, or simply the required mechanical accuracy or its tolerance (that defines the line uniformity) may pose unrealistic requirements for a practical realization.

We have therefore designed a high precision experiment aimed at proving both the dominant role of modal dispersion in long timing RPCs and the possibility of implementing a simple compensation technique (hereafter 'electrostatic compensation').

\subsection{Electrostatic compensation in 2-strip structures}

\subsubsection{Description of the experiment}

Several 2meter-long electrodes were specially manufactured, each consisting of 2 parallel $0.05 \mathrm{~mm}$-thick, $25 \mathrm{~mm}$-wide copper strips on an $0.25 \mathrm{~mm}$-thick epoxy glass laminate as substrate (G10). The strip width and interstrip separation were accurately defined within $\pm 0.1 \mathrm{~mm}$, as verified by microscope. ${ }^{10}$ Electrodes not fulfilling this condition were rejected for the experiment. ${ }^{11}$ The basic test structure was that of a micro-strip configuration with strips placed above a 1 or 2 -gap structure laying on a

\footnotetext{
${ }^{10}$ I.e, the standard deviation of the inter-strip distance as measured along the strips length is of the order of $100 \mu \mathrm{m}$.

${ }^{11}$ The acceptance yield was $4 / 20$.
} 
quasi-infinite ground plane (Fig. 4). ${ }^{12}$ Various inter-strip separations were essayed, but only $2.1 \mathrm{~mm}$ and $3.1 \mathrm{~mm}$ were systematically characterized. The definition of the gas gaps was performed through nylon monofilaments of $0.3 \pm 0.01 \mathrm{~mm}$ diameter interleaved on $1 \pm 0.05 \mathrm{~mm}$ float glass plates, ${ }^{13}$ arranged in the direction across the strips with a $5 \mathrm{~cm}$ pitch. We present first the measurements performed in the time domain and a separate discussion is devoted to high-precision frequency-domain measurements in order to assess losses (section 4). A pulser with rise-time $t_{\text {rise }}=280 \mathrm{ps},{ }^{14} \mathrm{FWHM} \Delta t=700 \mathrm{ps},{ }^{15}$ a repetition rate of $50 \mathrm{~Hz}$ and output impedance $R=50 \Omega$ was injected into one of the ports (1) and signals recorded in the opposite port (2), in the near-end (3) and in the far-end cross-talk ports (4), (see Fig. 4-down). The signal was split before injection with a $6 \mathrm{~dB}-2 \mathrm{GHz}$ signal divider and sent both to the electrode structure and to a $2.5 \mathrm{GHz}$ Tektronics scope at 10 Gsamples/s. Ten cm long $50 \Omega$ BNC cables terminated on $50 \Omega$ were attached both to the far-end of the structure and to the near-end cross-talk port (3). The electrical connection between those cables and the electrodes was performed over $1 \mathrm{~cm}$ length through flexible copper strips soldered with tin.

The uniformity of the line was ensured by applying weight on $10 \mathrm{~cm}$-thick blocks of extruded polystyrene foam via stainless steel bricks. The foam was attached directly to the strips. A high uniformity proved to be extremely important, since the very thin electrodes tended to bendup easily over $1 \mathrm{~mm}$ or more and break the line impedance, giving immediately very large cross-talk and dispersion patterns. We will ascribe the electrical behavior of this auxiliary ensemble to that of air, with $\epsilon_{r}=1$. We have evaluated both in experiment and simulation the effect of the foam thickness and the steel, and concluded that it can be effectively considered to behave electrically like air.

Measurements were stored when the waveforms at all ports were independent from additional pressure applied onto the strips and all connections had been checked. Compensation was simply achieved by placing additional glass plates above the electrodes.

\subsubsection{Measurements}

As indicated in Fig. 4, all the measurements performed in the following were done on a resistance $R=50 \Omega$. For better representation, the fraction of transmitted signal $F_{t r}(t)$ is defined as the ratio of the measured transmitted current $I_{t r}(t)$ to the maximum of the injected one $I(t)$. On the other hand, the fraction of cross-talk $F_{c t}(t)$ is defined as the ratio

\footnotetext{
$\overline{12}$ The width of the ground plane and glass plates was $60 \mathrm{~cm}$ and 50 $\mathrm{cm}$ respectively. According to MAXWELL-2D simulations (see later), they can be effectively considered to be infinite for the capacitance calculations.

${ }^{13}$ Schott.

${ }^{14}$ Defined as the time elapsed from a fraction 0.1 to 0.9 of the signal maximum.

${ }^{15}$ Full width at half maximum.
}

of the measured cross-talk current $I_{c t}(t)$, to the maximum of the transmitted one:

$$
\begin{aligned}
& F_{t r}(t)=I_{t r}(t) / \max [I(t)] \\
& F_{c t}(t)=I_{c t}(t) / \max \left[I_{t r}(t)\right]
\end{aligned}
$$

where ' $\max []$ ' denotes the maximum of the bracketed function. We still need to decide how to define the injected signal $I(t)$ from the measured voltage in the scope. It turns out that the following definition for the normalization of $I_{t r}(t)$ is very convenient:

$I(t)=2 \frac{V(t)}{R}$

Here $V(t)$ is the voltage pulse measured after the splitter. As shown in appendix, under equivalence 21 the transmitted signals correspond to the ones in the physical situation where a current $I(t)$ was induced at one of the detector ends, $y_{o}=D$. Therefore, the solutions of eq. 10 :

$\vec{I}_{T}(t)=\frac{\hat{T}}{2} \sum_{j=0}^{\infty}(1-\hat{T})^{j} \hat{M}\left(\begin{array}{l}M_{11}^{-1} I\left(t-\frac{(-1)^{j} D+2\lceil j / 2\rceil D}{v_{1}}\right) \\ M_{21}^{-1} I\left(t-\frac{(-1)^{j} D+2\lceil j / 2\rceil D}{v_{N}}\right)\end{array}\right)$

with $\vec{I}_{T}(t)=\left\{I_{t r}(t), I_{c t}(t)\right\}$ can be directly compared with pulser data. For that, the recipe to be followed is that $F_{t r}(t)$ from eq. 19 is normalized according to eq. 21.

We present in Fig. 5 the oscillograms for the two cases for which electrostatic compensation, $\Delta v / \bar{v}=0$, was achieved with the aforementioned prosaic procedure of placing additional glass plates above the readout strips. As shown in Fig. 5 up-left, for $2.1 \mathrm{~mm}$ inter-strip separation and 1 gas gap electrostatic compensation could be roughly achieved for 1 additional glass plate (red/dark line), and similarly for $3.1 \mathrm{~mm}$ inter-strip spacing in case of 2 gas gaps, downleft. The un-compensated systems show a distinct bipolarity with an additional difference in sign from the undercompensated (green/gray line) to the over-compensated (blue dashed line) one. On the other hand, the compensated systems show a factor 10 smaller cross-talk signal, having the same shape than the original one. Approximately equal levels are achieved in this case both for the far-end and near-end cross-talk (horizontal dashed line in Fig. 5). In any case, after just including the first reflection, the cross-talk has approximately zero net charge in any of these configurations. It approaches the expected zero value in the limit where all reflections are included (see appendix).

The overall behavior of the oscillograms is reasonably captured (Fig. 5-right) by a loss-less simulation based on eq. 22. Capacitance matrices were obtained via a Finite Element Method (FEM) calculation from the MAXWELL2D package [38]. A relative dielectric constant for float glass of $\epsilon_{r}=5.5$ was used, acccording to a direct measurement presented in section 4.1. For G10, a typical value $\epsilon_{r}=4.4$ was chosen. The time-offsets present in the oscillograms due to cables were substracted in order to match the simulated 
1-gap, $2.1 \mathrm{~mm}$ inter-strip separation

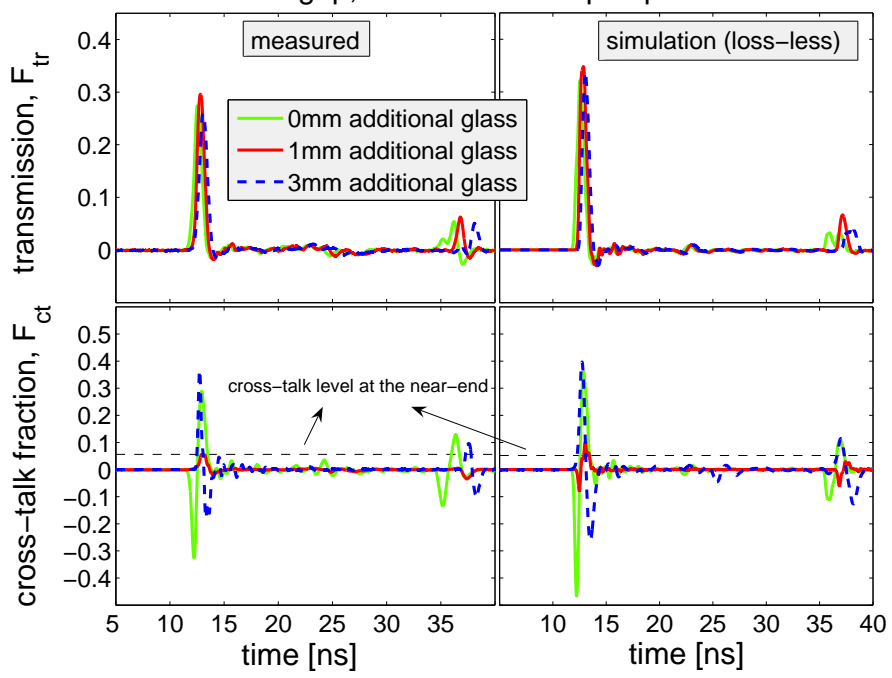

2-gaps, $3.1 \mathrm{~mm}$ inter-strip separation

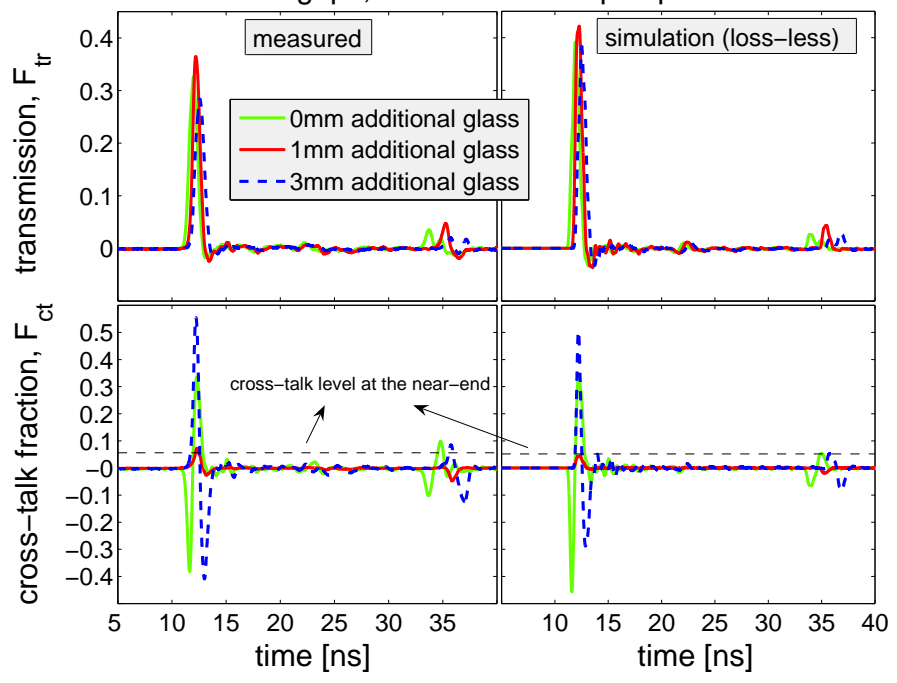

Fig. 5. Up-set: fraction of transmitted signal and cross-talk fraction for a 2-strip RPC with $2.1 \mathrm{~mm}$ inter-strip separation and 1 gas gap, for an under-compensated case (green/dark line), compensated (red/dark line) and overcompensated (dashed blue line). Low-set: as in the upper set, but for $3.1 \mathrm{~mm}$ inter-strip separation and 2 gas gaps. Up to a factor 10 cross-talk suppression can be achieved if the system is adequately compensated. The far-end and near-end cross-talk become equal in that case (horizontal dashed line). Figures to the right show simulations assuming the structure to be loss-less and taking the measured value $\epsilon_{r}=5.5$ for float glass. Capacitance matrices calculated with MAXWELL-2D.

waveforms. Besides this fact, the simulations are therefore parameter-free. ${ }^{16}$

Despite the apparent close agreement in Fig. 5, in order to perform an adequate evaluation we have followed a more application-oriented approach: i.e., when aimed at a precise time determination at high efficiency in a multi-hit

\footnotetext{
${ }^{16}$ A full Finite Difference Time Domain (FDTD) solution of the telegrapher's equations performed with the APLAC HF-simulator [33], used in [2], was also attempted. The calculation shows, however, numerical instabilities due to the presence of a large ground electrode and is being currently investigated.
}

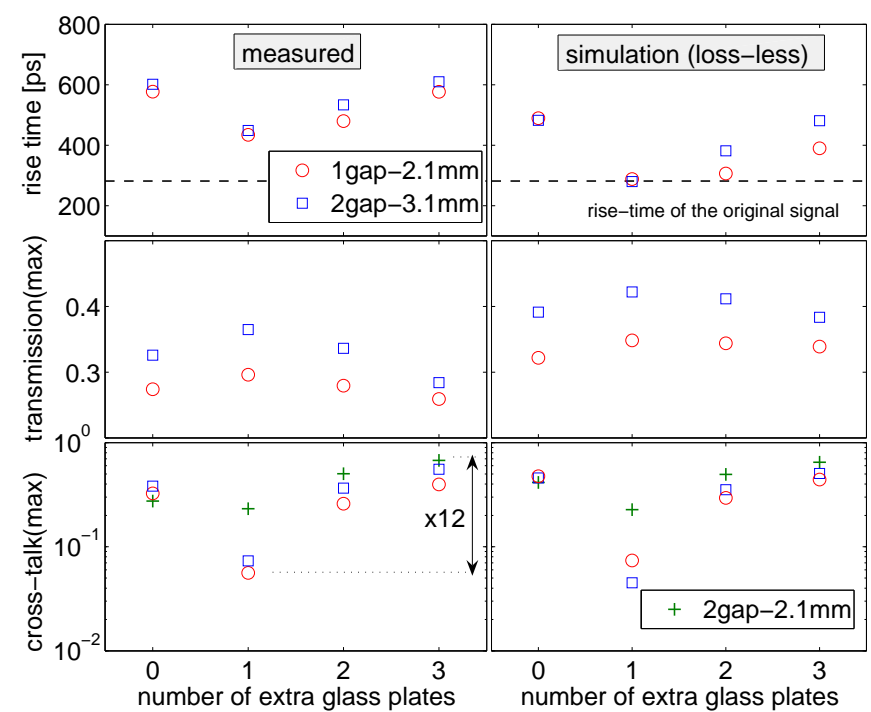

Fig. 6. Left column: measured signal rise-time, maximum fraction of transmitted signal and maximum fraction of cross-talk for 2.1 and $3.1 \mathrm{~mm}$ inter-strip separation for 2 -strip structures with 1 (o) and $2(\square)$ gaps, respectively. Right column: the same observables as obtained from a parameter-free simulation neglecting losses. The compensated system ( $\sim 1$ additional $1 \mathrm{~mm}$-thick glass plate) clearly shows the most favorable properties. The 2 gap- $2.1 \mathrm{~mm}$ case $(+)$ is shown for further insight (compensation could not be achieved since it requires a glass thickness in between 0 and $1 \mathrm{~mm}$, not available during the measurements). Cross-talk can be suppressed up to a factor of 12 by simple design choices.

environment the main figures of merit of a multi-strip system are, probably: i) the deterioration of the signal risetime during transmission, ii) the maximum transmitted signal $\left(\max \left[\left|F_{t r}(t)\right|\right]\right)$ and iii) the maximum crosstalk fraction $\left(\max \left[\left|F_{c t}(t)\right|\right]\right)$. These quantities are compiled in Fig. 6 for measurements (left) and simulations (right) for various structures, as a function of the number of additional glass plates. The system clearly exhibits more favorable properties when it is compensated, showing a higher transmission, lower shaping and minimal cross-talk. The smaller measured transmission and larger signal rise-times as compared to simulations could be traced back to losses in the line and are discussed in detail in next section. It may look like a small effect but the $\sim 200$ ps offset observed in data with respect to simulations in Fig. 6 up-left is about a factor of two higher than the intrinsic signal rise-times expected for RPC signals in the absence of Space-Charge, $t_{\text {rise }}=$ $\ln 9 / S \simeq 110$ ps. A precise simulation of transmission patterns can be thus attempted only after including losses and is given in the next section.

Crosses (+) in Fig. 6-down are aimed at highlighting that compensation is not related solely to the usage of an additional $1 \mathrm{~mm}$-thick glass plate (for $2.1 \mathrm{~mm}$ inter-strip separation and 2gaps compensation would require of $\simeq 0.5 \mathrm{~mm}$ glass thickness, not available during the experiment). By looking at the cross-talk patterns it is clear that the precision required for this system to be compensated is well below $1 \mathrm{~mm}$. Precisely, $1 \mathrm{~mm}$ difference, either in the addi- 
tional compensating glass or in the inter-strip distance, can easily imply a cross-talk difference of up to a factor of $\times 10$, together with a worsening in the signal rise-time by $200 \mathrm{ps}$.

\subsubsection{The 2-strip parameters}

The '2-strip parameters' contain all the information necessary for characterizing a loss-less 2-strip transmission line. They are shown in Fig. 7, as derived from the capacitance matrices obtained from MAXWELL-2D. Closed symbols indicate the exact values of the parameters while open symbols (almost indistinguishable), show the values obtained under the 'low-coupling' approximation (eqs. 1618).
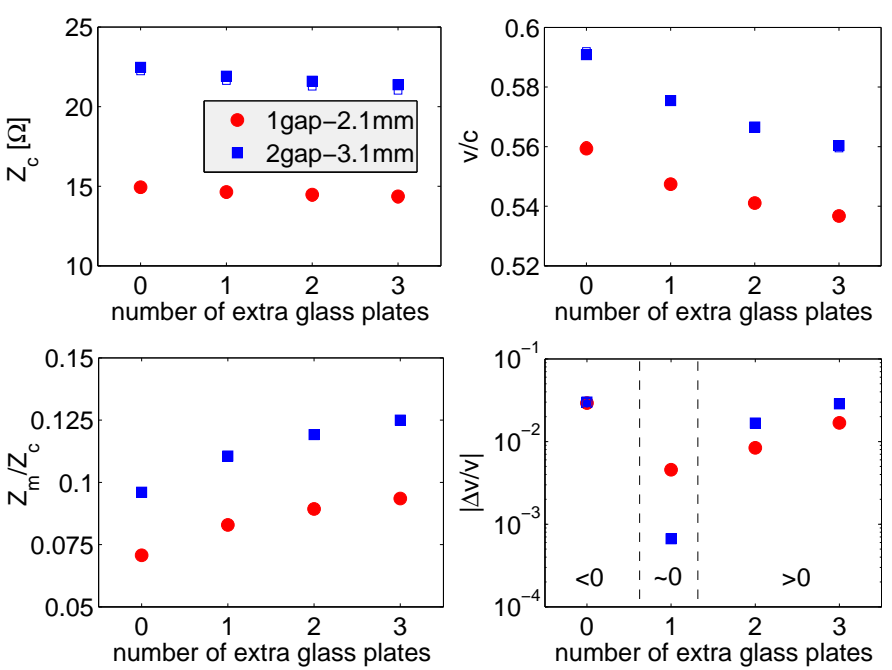

Fig. 7. Simulated '2-strip parameters' for different number of gaps and two different inter-strip separations, from up-left to down-right: characteristic impedance, propagation velocity, coupling coefficient and velocity dispersion (absolute value). Values obtained from a FEM calculation performed with the MAXWELL-2D solver. Closed symbols show the exact value and open ones (almost undistinguishable) the value under the 'low-coupling' approximation (eqs. 16-18).

Note that the coupling coefficient increases steadily with the number of additional glass plates, as intuitively expected, while the velocity dispersion shows a shallow minimum for 1 glass plate, roughly corresponding to a compensated situation. The other parameters have a very smooth dependence with the number of glass plates and clearly lack of importance for the phenomena here addressed.

\subsubsection{Solutions in the frequency domain for a loss-less 2-strip line}

Fig. 8 shows the simulated moduli of two scattering matrix parameters $S_{21}$ (transmission) and $S_{41}$ (far-end crosstalk) as obtained from the Fourier transform of the timedomain solutions given in eq. 10 (for details see next section). The base structure has an inter-strip separation of $3.1 \mathrm{~mm}$ and 2-gap/3-glass (as in Fig. 5-down) for which the following cases were studied: a) un-compensated (no additional glass) but un-matched, b) compensated $(1.05 \mathrm{~m}$ additional glass) but un-matched, c) un-compensated but matched, d) compensated and matched. Note that a 2strip system requires of 3 resistors in order to be perfectly matched, typically two in series with the ports and one in parallel between ports on the same strip end ( $R=24 \Omega$ and $R_{m}=195 \Omega$ here, respectively). The oscillatory pattern observed in Fig.8(up-right) is responsible for the reflections observed in the counter while Fig.8(down-left) indicates the pure effect of modal dispersion. The characteristic bandstop region at approximately $1 \mathrm{GHz}$ was first predicted and measured in micro-strips by G. I. Zysman and A. K. Johnson as early as 1969 [34]. ${ }^{17}$ It must be noted that a homogeneous 2-strip loss-less system has a flat frequency response (no signal shaping) if its impedance is matched while an inhomogeneous one requires, additionally, to be electrostatically compensated.
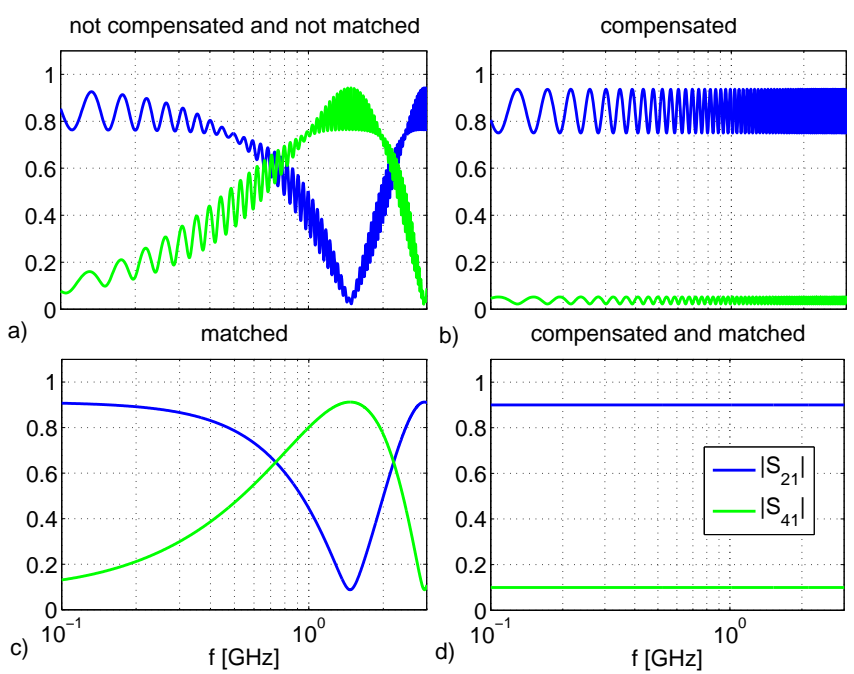

Fig. 8. Simulated moduli of the transmission $S_{21}$ and far-end cross-talk $S_{41}$ coefficients for 4 exemplary cases in a 2 -gap/3-glass RPC with $3.1 \mathrm{~mm}$ inter-strip separation. From up-left to down-right: a) un-compensated and without impedance matching, b) compensated but without matching, c) un-compensated but matched and d) compensated and matched. No shaping is thus expected for the latter case, under the assumption of loss-less line.

\section{Deviation from the loss-less TEM propagation mode}

Losses have been neglected for the derivation of eq. 10 and Fig. 8 and have been excluded from previous analysis [21]. There are two main sources of losses in a transmission line:

On the one hand it stands the finite resistance of the readout strips at high frequency, since conduction is then confined to a very thin layer ('skin effect'). Assuming that conduction takes place along $75 \%$ of a skin-depth (typical value, $[3]$ ), the resistance per unit length of a strip of thickness $t \ll \mathrm{w}$ is, at high frequencies:

\footnotetext{
${ }^{17}$ In printed circuit design, micro-strips are usually inhomogeneous, unlike strip-lines.
} 
$R \simeq \sqrt{\frac{\pi}{2} \mu \rho_{D C}} \times \frac{\sqrt{f}}{\mathrm{w}}$

where $\rho_{D C}$ is the DC conductivity of the material considered, and $\mu$ its magnetic permeability.

On the other hand, losses can be originated due to the shunting conductance between the strips themselves and/or between strips and ground. Being usually very well insulated electrically (in RPCs either glass or Bakelite can be effectively considered as ideal insulators for the sake of signal transmission), the shunting conductance is expectedly governed by the dielectric losses on the insulator materials:

$G_{\text {glass }}=\left.2 \pi C_{\text {glass }} \tan \delta\right|_{\text {glass }} \times f$

The loss-tangent $\left.\tan \delta\right|_{\text {glass }}$ is the ratio of the imaginary to the real part of the dielectric constant of the given medium and $C_{\text {glass }}$ is its capacitance with respect to ground (assumed to be glass here). The losses due to either the polarization of air or the standard gas mixture will be neglected in the following due to their very low density of electric dipoles. However, even in this simple case, an analytical evaluation of the losses becomes complicated in general for an inhomogeneous structure. A practical approach to perform this calculation is given in [3], by considering that the (now) complex capacitance is given, to a good approximation, by the series capacitance of the system (no edge effects). In such a case a simple parallel plate capacitor formula can be used and, after some simple algebra, the conductance for the whole structure can be estimated as:

$$
\begin{aligned}
& \left.G \simeq 2 \pi C_{g} F \tan \delta\right|_{\text {glass }} \times f \\
& G \simeq F G_{\text {glass }}, \text { with } \quad F=\frac{C_{g}}{C_{\text {glass }}} \\
& \frac{G}{G_{\text {glass }}} \simeq \frac{C_{g}}{C_{\text {glass }}}
\end{aligned}
$$

where $C_{g}$ is the capacitance with respect to ground of the whole structure. As intuitively expected, the shunting conductance is thus reduced by interleaving gas. We will define the effective loss-tangent of the structure as $\tan \delta^{*}=$ $\left.F \tan \delta\right|_{\text {glass }}$.

Losses are usually discussed in a frequency-domain representation, since they are expected to have a very characteristic dependence $(23,24)$. Losses can be experimentally determined through a 1-strip transmission measurement. For this it is useful to make use of the fact that the complex transmission coefficient from port 1 to 2 has the simple analytical expression [3]:

$$
S_{21}(f)=\frac{(2-T) T}{1-(1-T)^{2} e^{-2 \gamma D}} e^{-\gamma D}
$$

For completeness, the reflection coefficient $S_{11}$ is given as:

$S_{11}(f)=1-T \frac{1+(1-T) e^{-2 \gamma D}}{1-(1-T)^{2} e^{-2 \gamma D}}$

The transmission coefficient $T=2 Z_{c} /\left(R+Z_{c}\right)$ is now a complex number, derived from the complex impedance $Z_{c}$ :
$Z_{c}=\sqrt{\frac{R+j 2 \pi f L_{0}}{G+j 2 \pi f C_{g}}}$

and

$\gamma=\sqrt{\left(R+j 2 \pi f L_{0}\right)\left(G+j 2 \pi f C_{g}\right)}=\frac{1}{\Lambda}+j \beta$

The reflection and transmission coefficients verify, in a loss-less system, the condition $\left|S_{11}\right|^{2}+\left|S_{21}\right|^{2}=1$.

Although the exact formulas $28-31$ will be used in the following for the sake of precision, the 'low-loss' approximation $\frac{G}{2 \pi f C_{g}}, \frac{R}{2 \pi f L_{0}} \ll 1$ is often used because at high frequencies it is fulfilled for most practical purposes. ${ }^{18} \mathrm{Un}$ der this approximation additional insight can be obtained since:

$\frac{1}{\Lambda} \simeq \frac{1}{\Lambda_{G}}+\frac{1}{\Lambda_{R}}$

being:

$$
\begin{aligned}
& \Lambda_{R} \simeq \frac{2 Z_{c}}{R} \\
& \Lambda_{G} \simeq \frac{2}{G Z_{c}}
\end{aligned}
$$

and $\beta$ equals:

$\beta \simeq \frac{2 \pi f}{v}=2 \pi f \sqrt{L_{0} C_{g}}$

As it can be readily obtained from eqs. $23,25,33,34$ the geometrical dependence of $\Lambda_{R}, \Lambda_{G}$ with w is canceled in first order for wide-strip RPCs (w $\left.\gg h \rightarrow\left(Z_{c}, C\right) \sim 1 / \mathrm{w}\right)$. So, in practice, the main variables ruling the losses are indeed the frequency, the propagation distance and the loss-tangent. The cutoff frequency for each of the two processes 33, 34 can be obtained approximately from the dominant $e^{-\frac{D}{\Lambda}}$ behavior in eq. 28, yielding:

$$
\begin{aligned}
& f_{c, R} \simeq\left(\frac{Z_{c} \mathrm{w} \ln 2}{D}\right)^{2} \frac{2}{\pi \mu \rho_{D C}} \\
& f_{c, G} \simeq \frac{v \ln 2}{2 \pi D \tan \delta^{*}}
\end{aligned}
$$

\subsection{Losses in 1-strip structures}

We characterized the float glass employed by measuring its frequency response to transmission along a $2.5 \mathrm{~cm}$-wide strip. Measurements were done with a 4-port network analyzer ( $3.5 \mathrm{GHz}$ bandwidth) with the strip placed along a $2 \mathrm{~m}$ long glass stack placed over a quasi-infinite ground plane (like in previous section), but we performed a control measurement by placing it transversally $(0.50 \mathrm{~m}$ length) and so reducing the losses. The space between strip and ground was filled with three $1 \mathrm{~mm}$-thick glass plates, and pressure

\footnotetext{
${ }^{18}$ For a system dominated by dielectric losses this condition translates simply into $\tan \delta^{*} \ll 1$, that is the case here as we will see, but also a rather typical situation for most dielectric materials.
} 
was applied via polystyrene foam as described in previous section. Additional glass plates were placed above for evaluating possible systematic errors; we also compared the results for a G10-supported strip with the ones for standard $\mathrm{Cu}$ tape of the same width. In all cases the differences were minimal. Fig. 9 shows the measured and simulated modulus of the transmission coefficient $S_{21}$ for a G10-supported strip and no additional glass plate. The electrostatic parameters $C_{g}$ and $L_{0}$ were obtained from MAXWELL-2D. The best overall description implies a value for $\epsilon_{r}=5.5$ for the glass, that describes very well the inter-peak separation $\Delta f=v /(4 \pi D)$ and the amplitude oscillations at low frequencies (see insets). A close look at this observable allows to determine that the dielectric constant is varying by $5 \%$ at most in the range $f=[0.1-1] \mathrm{GHz}$. At higher frequencies, the observed behavior is dominated by the dielectric losses.

We tried to describe the transmission at high-frequencies by assuming a constant value of the loss-tangent $\tan \delta=$ 0.021 , that provides a reasonable description of the data. It over-estimates, however, the signal attenuation at low frequencies. The data favors a soft increase with frequency in the range $[0.1-3.5] \mathrm{GHz}$, that we have operationally parameterized with a logarithm (table 1), for simplicity:

$\tan \delta(f[\mathrm{GHz}])=\tan \delta_{0.1}+\frac{\tan \delta_{3}-\tan \delta_{0.1}}{\log _{10} \frac{3}{0.1}} \log _{10} \frac{f}{0.1}$

Such a smooth increasing behavior at ambient temperature is qualitatively compatible with the one reported in [39].

Table 1

\begin{tabular}{|c|c|c|c|c|}
\hline & $\tan \delta_{0.1}$ & $\tan \delta_{3}$ & $\tan \delta$ & $f_{c}[\mathrm{GHz}]$ \\
\hline $\mathrm{D}=2 \mathrm{~m}$, only glass & 0.007 & 0.021 & 0.021 & 0.55 \\
\hline $\mathrm{D}=0.5 \mathrm{~m}$, only glass & 0.007 & 0.032 & 0.03 & 1.3 \\
\hline $\mathrm{D}=2 \mathrm{~m}, 2$-gaps $/ 3$-glass & 0.007 & 0.032 & 0.029 & 0.85 \\
\hline
\end{tabular}

Table with the best description of the observed losses for three different structures. The first two columns show the 2 parameters obtained by assuming a logarithmic increase with frequency in the range $f=[0.1-3.5] \mathrm{GHz}$ (eq. 38). The third column shows the best description assuming a constant value. The last column shows the cutoff frequency for each structure.

Apart from the measurements for a $3 \mathrm{~mm}$-thick $2 \mathrm{~m}$-long stack, additional measurements on an $0.5 \mathrm{~m}$-long stack and a 2-gap/3-glass structure were performed, and the best values obtained for the $\tan \delta$ of the glass are given in table 1 . The third column shows the value for $\tan \delta$ when assumed to be constant over the whole frequency range, from which an average value $\tan \delta=0.25 \pm 0.05$ in the range $\mathrm{f}=[0.1$ 3.5] GHz can be inferred for the float glass we used, dominated by systematic uncertainties. The cutoff frequency $f_{c}$ is also given in the last column. Due to the highly oscillatory pattern, it was determined from a comparison with the simulated $\left|S_{21}\right|$, as in Fig. 9. The value of $f_{c}$ was then obtained through evaluation of the condition $e^{-D / \Lambda}=1 / \sqrt{2}$.

Note that the cutoff frequency is as small as $f_{c}=$ $0.55 \mathrm{GHz}$ for the propagation over a $3 \mathrm{~mm}$-thick glass stack
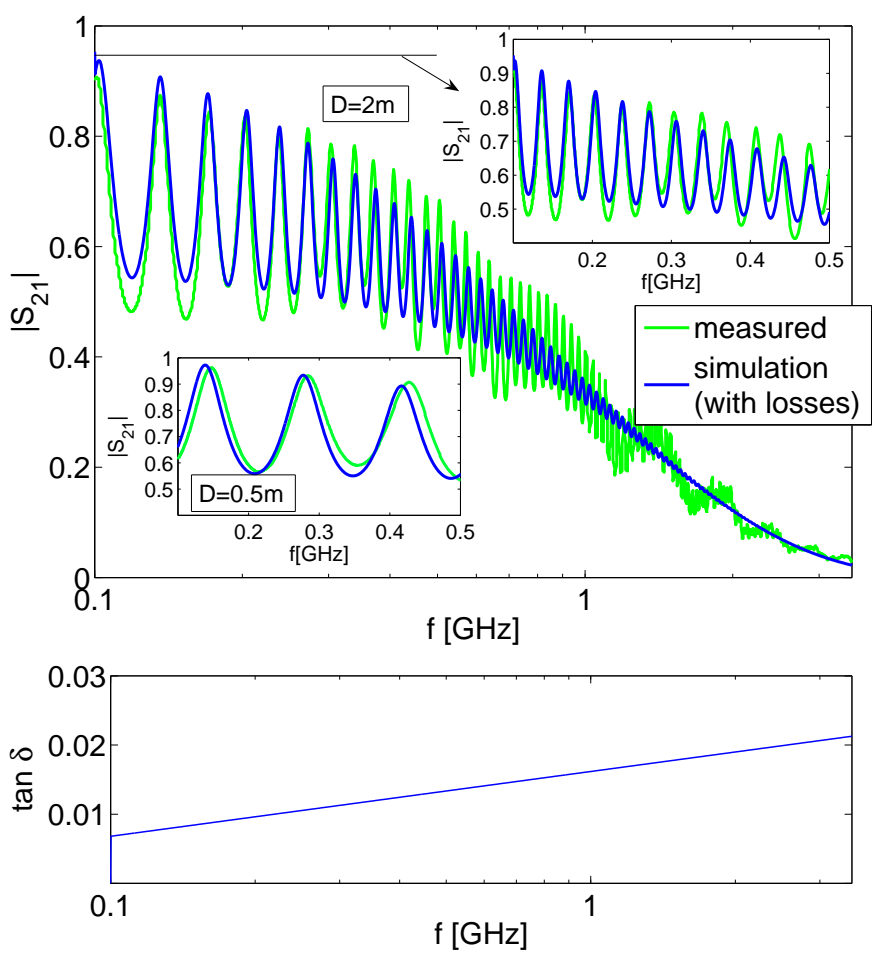

Fig. 9. Up: modulus of the transmission coefficient $S_{21}$ in a $2 \mathrm{~m}$-long strip placed above a $3 \mathrm{~mm}$-thick glass stack both for measurements (green/gray) and simulations including losses (blue/dark). The inter-peak distance provides a value for $\epsilon_{r}=5.5$ within $5 \%$ in the range $\mathrm{f}=[0.1-1] \mathrm{GHz}$ (upper inset, up to $0.5 \mathrm{GHz}$ ). The lower inset shows the same measurement along $50 \mathrm{~cm}$ length (the same stack but with the strip rotated). Down: a functional description of the loss-tangent that provides a good agreement with transmission measurements above $0.5 \mathrm{GHz}$.

along $2 \mathrm{~m}$. The situation improves when including gas gaps, up to $f_{c}=0.85 \mathrm{GHz}$, but still far from the intrinsic cutoff frequency $f_{c}=3 \mathrm{GHz}$ expected for RPC signals. Transmission is indeed limited essentially by the dielectric losses in the glass since the attenuation due to resistive losses is as small as $1 / 1.05$ at $3 \mathrm{GHz}$. As said, the dependence of $\Lambda_{G}$ and $\Lambda_{R}$ with the particular geometry is very small for wide-strip RPCs so skin effect will play usually a minor role in this type of detectors.

\subsection{Deviation from quasi-TEM propagation}

The dumped oscillating behavior above $1 \mathrm{GHz}$ in Fig. 9, overlaid on the pure system losses can not be accommodated in a simple quasi-TEM image, and is indeed present for all measurements on $2 \mathrm{~m}$-long/0.6m-wide structures. This discrepancy can be highlighted by studying the sumcoefficient $S=\sqrt{\left|S_{21}\right|^{2}+\left|S_{11}\right|^{2}}$, that is shown in Fig. 10. Measurements (green/gray line), simulations assuming losses (blue/dark line) and without losses $(|S|=1$, dashed) are plotted for two cases: in Fig. 10-up the transmission is measured along $2 \mathrm{~m}$ over an $0.6 \mathrm{~m}$-wide ground plane while in Fig. 10-down the transmission is measured on the rotated structure, meaning an $0.5 \mathrm{~m}$-long strip over a $2 \mathrm{~m}$ - 
wide ground plane, the later showing a better agreement with simulations.

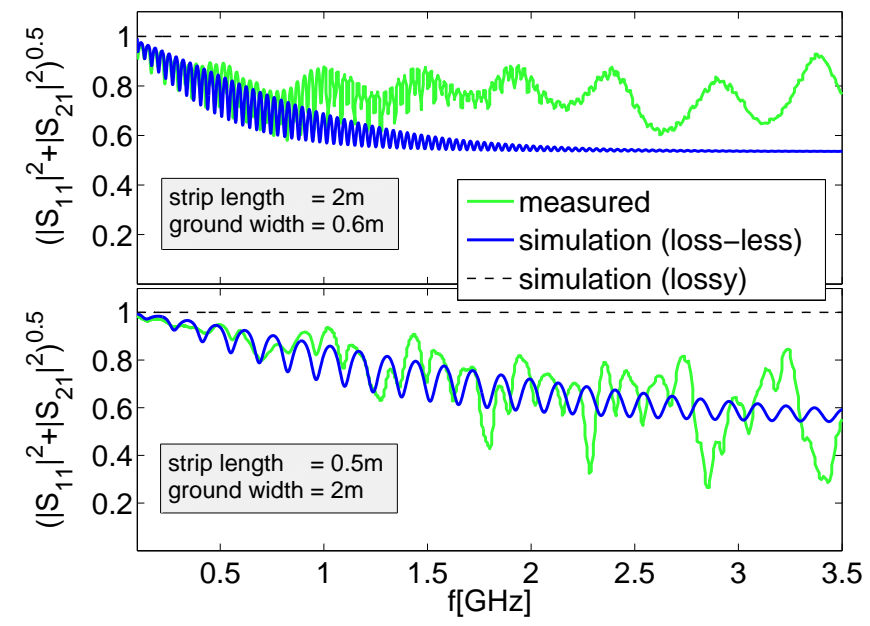

Fig. 10. Up: Sum-coefficient $S=\sqrt{\left|S_{21}\right|^{2}+\left|S_{11}\right|^{2}}$ for measurements (green/gray), simulations assuming losses (blue/dark), and simulations without losses $(|S|=1$, dashed). Down: the same as above but with the strip rotated (ground plane approximately $\times 4$ wider). Deviations from a pure TEM description are suppressed in the lower case, due to the ground plane being closer to the ideal infinite limit.

It must be noted that the critical frequency for the lowest TE (transverse electric) mode to start propagating along a strip placed over an infinite ground plane at close distance from it is:

$f_{T E}>\frac{v}{2 \mathrm{w}}$

where $\mathrm{w}$ is the width of the strip, thus yielding $f_{T E}>$ $7 \mathrm{GHz}$. The frequency at which simulations and measurements deviate in Fig. 10-up is, however, $f_{T E} \simeq 0.3 \mathrm{GHz}$, corresponding to a width of $\simeq 60 \mathrm{~cm}$, exactly as our ground plane. Incidentally, measurements in Fig. 10-down over the rotated structure $\left(f_{T E} \simeq 0.075 \mathrm{GHz}\right)$ show a much better agreement with a quasi-TEM description based uniquely on eqs. 28,29 . In order to better understand this fact, it must be recalled that propagation of non-TEM modes is theoretically forbidden below the cutoff frequency when the widths of both planes (strip and ground) satisfy simultaneously condition 39 (see [40], for instance). Since an infinite ground plane mimics the presence of a mirror strip at equal distance from it, the same condition applies for this latter case, as long as the plane is infinite in extension. This would explain the better agreement with a TEM-description for a wider ground plane, as in Fig. 10-down. In any case, it will be shown in the next section that these deviations from pure quasi-TEM propagation do not need to be included in order to accurately reproduce the typical figures of interest in RPC transmission.

\subsection{Losses in 2-strip structures}

Unfortunately, the solutions to the Transmission Line problem including losses become immediately non-analytic for more than 1 strip [41]. Our proposal is to assume that the eigen-vectors and eigen-values of the problem are very slightly modified in the presence of losses, so that the structure of the solutions remains the one provided by the lossless problem, and losses can be included as a convolution at a later stage. Additionally, the cross-conductance between strips is neglected. In order to experimentally demonstrate this assertion, measurements on 2-strip structures were performed, and simulations carried out under this factorization assumption. We first need to adapt the solutions given by eq. 10 to the case where measurements are performed with a network analyzer. For this we recall that the network analyzer was operated with standard terminations $(R=50 \Omega)$ and that eq. 10 can be easily adapted to this particular case, similarly to previous section. The array of voltages measured at the far-end in the time domain for a voltage source $V(t)$ (as measured on $R=50 \Omega$ ) connected to strip $n$ can be then obtained as:

$$
\vec{V}_{F E}(t)=\hat{T} \sum_{j=0}^{\infty}(1-\hat{T})^{j} \hat{M}\left(\begin{array}{c}
M_{1 n}^{-1} V\left(t-\frac{(-1)^{j} D+2\lceil j / 2\rceil D}{v_{1}}\right) \\
\ldots \\
M_{N n}^{-1} V\left(t-\frac{(-1)^{j} D+2\lceil j / 2\rceil D}{v_{N}}\right)
\end{array}\right)
$$

That is nothing else but eq. 22 for N-strips under the equivalence 21 . The calculation of the voltages at the nearend $\vec{V}_{N E}(t)$ is analogous, but requires the incoming voltage pulse to be explicitely considered at the given port:

$$
\begin{aligned}
& \vec{V}_{N E}(t)=\hat{T} \sum_{j=0}^{\infty}(1-\hat{T})^{j} \hat{M} \times \\
& \times\left(\begin{array}{c}
M_{1 n}^{-1} V\left(t-\frac{D-(-1)^{j} D+2\lfloor j / 2\rfloor D}{v_{1}}\right) \\
\ldots \\
M_{N n}^{-1} V\left(t-\frac{D-(-1)^{j} D+2\lfloor j / 2\rfloor D}{v_{N}}\right)
\end{array}\right)-\left(\begin{array}{c}
\ldots \\
0_{n-1} \\
V(t) \\
0_{n+1} \\
\ldots
\end{array}\right)
\end{aligned}
$$

The scattering matrix parameters can be obtained by directly computing the transmission at different frequency components through a Fourier transform:

$$
\vec{S}_{F E, N E}(f)=\frac{\mathrm{ft}\left(\vec{V}_{F E, N E}(t)\right)}{\mathrm{ft}(V(t))}
$$

This equation provides, indeed, the solutions to the loss-less situation. Our proposal for including losses is the ansatz:

$$
\left.\vec{S}_{F E, N E}(f)\right|_{\text {lossy }}=\left.\vec{S}_{F E, N E}(f)\right|_{\text {loss-less }} \times \exp \left(-\frac{D}{\Lambda(f)}\right)
$$

that, translated into the time-domain, effectively implies a convolution of the solutions in eq. 10,40 or 41 with the in- 
verse Fourier transform of $\exp \left(-\frac{D}{\Lambda(f)}\right)$. As can be deduced from the functional dependence of $S_{11}$ (eq. 29) this ansatz is not accurate for the reflection coefficient. This is fortunately not a relevant parameter to understand transmission of signals induced inside a counter, so it is not considered further. Thus, by using the port numbering scheme in Fig. 4:

$\left.S_{2,3,41}(f)\right|_{\text {lossy }}=\left.S_{2,3,41}(f)\right|_{\text {loss-less }} \times \exp \left(-\frac{D}{\Lambda(f)}\right)$

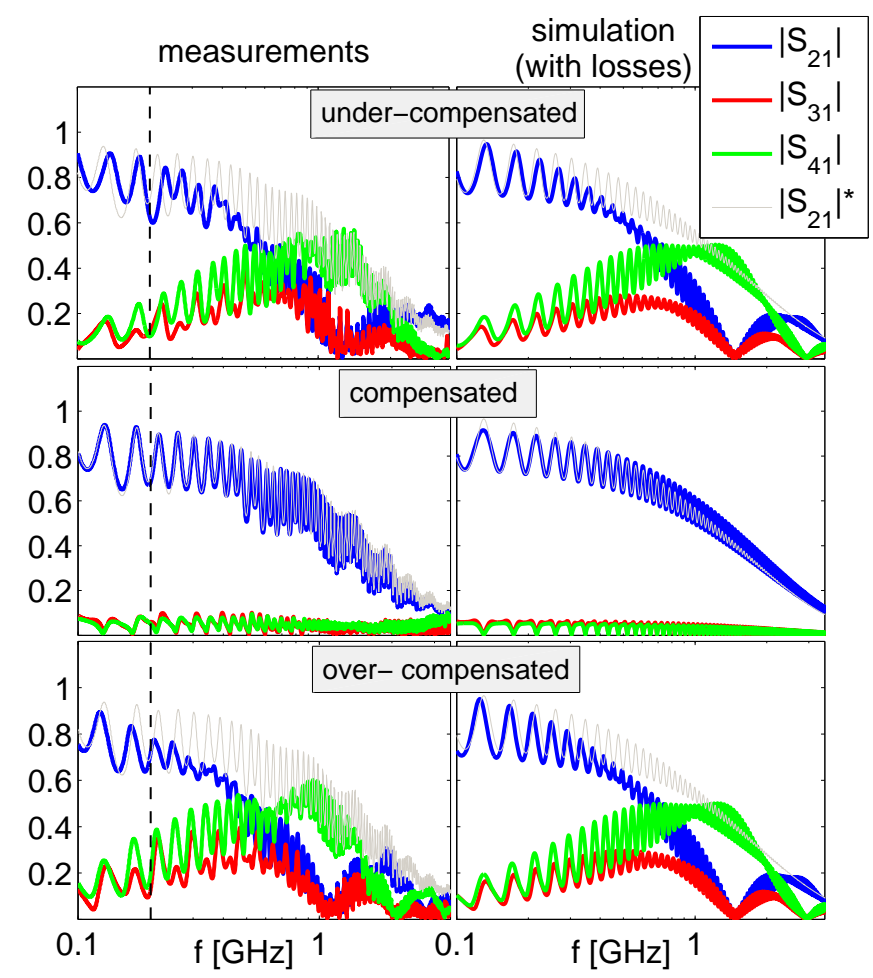

Fig. 11. Left: the measured moduli of the transmission coefficient $S_{21}$, far-end cross-talk $S_{41}$ and near-end cross-talk $S_{31}$ in a 2-gap/3-glass structure, with 2 strips separated by $3.1 \mathrm{~mm}$. From up to down, an under-compensated case (no additional glass), compensated (one additional glass plate) and over-compensated (three additional glass plates). $S_{21}^{*}$ is the transmission coefficient for 1-strip in the, otherwise, same structure. Right: simulations for the general lossy case under the factorization ansatz proposed in text. The vertical line shows the range previously measured in [21].

In order to numerically obtain the scattering matrix parameters we have assumed an initial voltage $V(t)$ with fast enough components in the range studied here. For simplicity, an exponential signal with 100ps rise-time has been used. The procedure was:

(i) Obtain the analytical solutions to the problem (eqs. 40, 41).

(ii) Make the Fourier transform, according to eq. 42.

(iii) Apply the attenuation factor $\exp \left(-\frac{D}{\Lambda(f)}\right)$ obtained from simulations of the 1-strip transmission coefficient $S_{21}$ as in Fig. 9.

Fig. 11 shows the measurements of the scattering matrix coefficients (left) and the corresponding simulations (right) for the 2-gap/3-glass structure previously studied in Fig. 5 in a time-domain representation. The 1-strip transmission coefficient is shown as $S_{21}^{*}$ (light grey) for reference. From up-down three cases are presented: under-compensated (no additional glass plate), compensated (one additional glass plate) and over-compensated (three additional glass plates). We note that the observed frequency pattern of the compensated system is strikingly simple, as predicted in Fig. 8, allowing for an extended bandwidth (up to the limit imposed by losses) and much reduced cross-talk patterns.

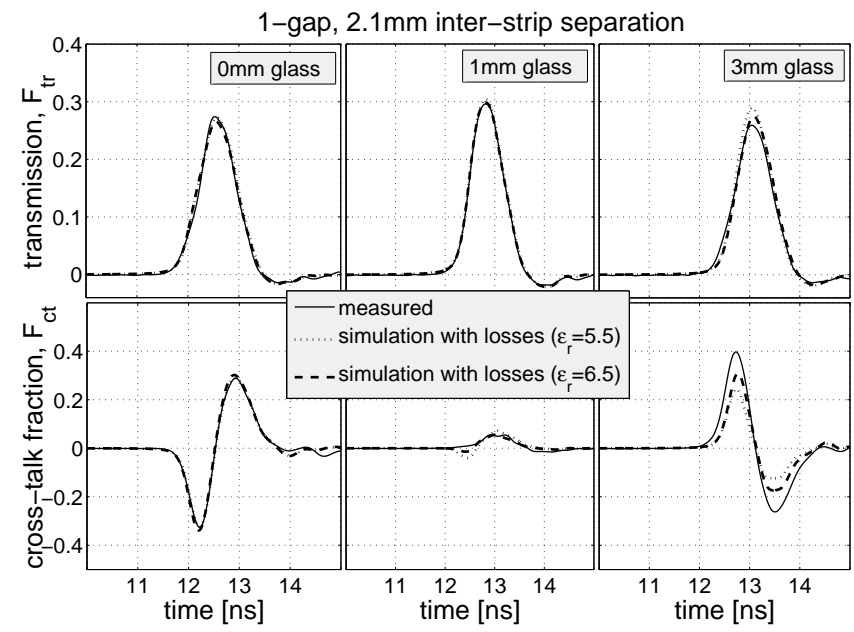

2-gaps, 3.1mm inter-strip separation

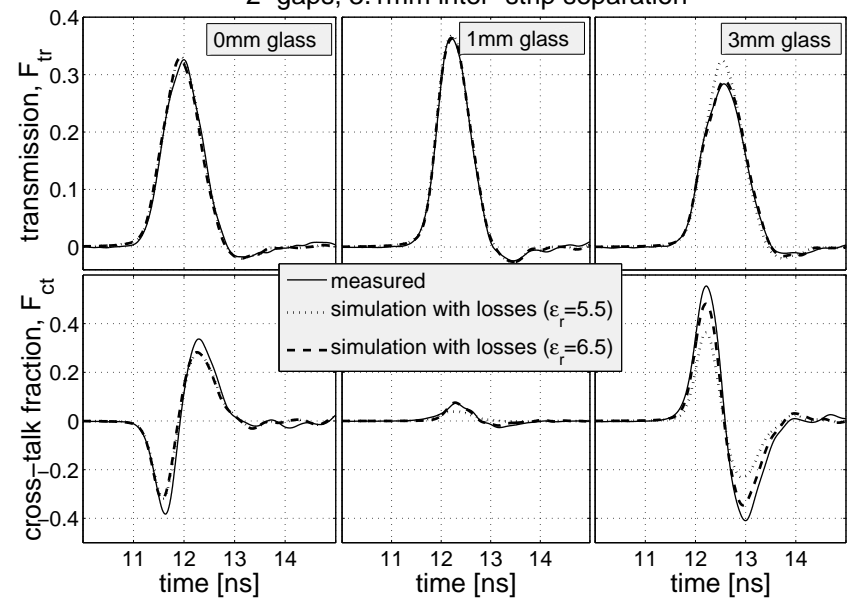

Fig. 12. Detailed comparison between full simulations including losses (dashed, dot-dashed) and the measured oscillograms (continuous lines). The comparison is made in the reflection-free region for the 1-gap RPC structure (up) and the 2-gap one (down) previously discussed in Fig. 5. From left to right, the cases 'under-compensated' (no additional glass), 'compensated' (1mm additional glass) and 'over-compensated' (3mm additional glass) are shown. Although a parameter-free simulation based on the directly measured value of $\epsilon_{r}=5.5$ provides a reasonable agreement, a value $\epsilon_{r}=6.5$ gives the best overall description.

In order to reconcile the measurements in frequency domain in Fig. 11 with the ones in time-domain in Fig. 5 we have convoluted (under the factorization assumption 44) the time-domain simulated waveforms in previous section with the inverse of the Fourier transform of $\exp \left(-\frac{D}{\Lambda(f)}\right)$. The results are shown in Fig. 12 by zooming-in the direct signal (no reflections). Time-offsets have been adjusted in 
order to allow precise shape-comparisons, for which the simulations with $\epsilon_{r}=5.5$ (dotted) are used as reference, and both measurements (continuous) and simulations with $\epsilon_{r}=6.5$ (dashed) are time-shifted to give the best possible agreement. A detailed comparison of the most relevant observables is given in Fig. 13 .

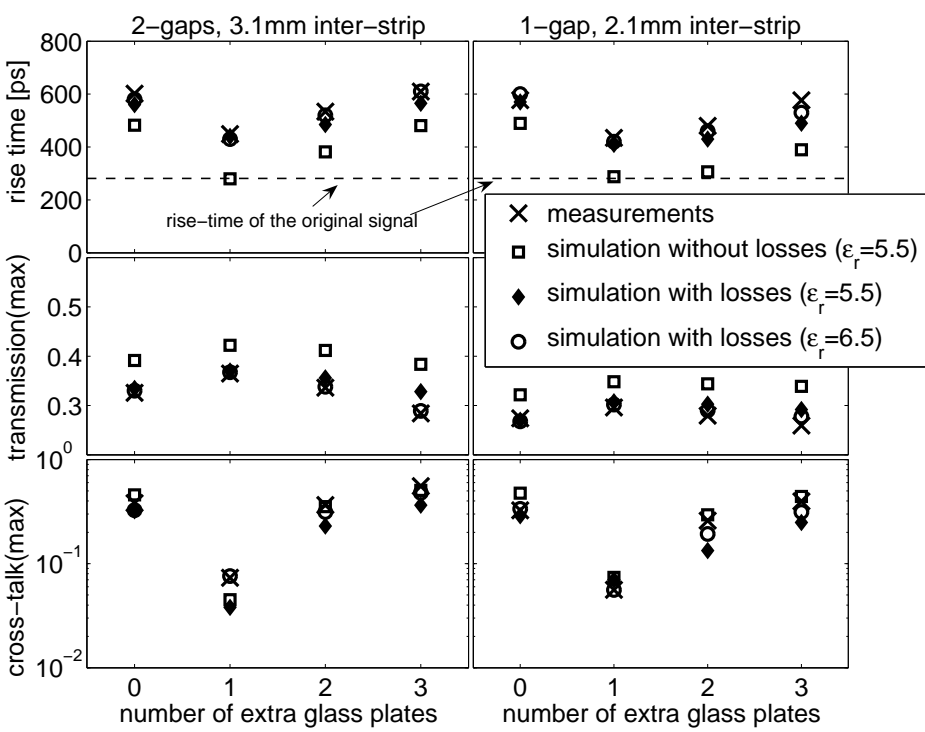

Fig. 13. Comparison between measurements and simulations for the main characteristics of a typical signal (signal rise-time, maximum of the signal transmission and cross-talk fraction). The signal characteristics are very well described after including losses, where the value $\epsilon_{r}=6.5$ for glass can be considered as an optimum choice.

Despite the overall good agreement, simulations clearly favor a value for $\epsilon_{r}=6.5$, contrary to the measurements in the frequency domain, that favor $\epsilon_{r}=5.5$. Of course, by using $\epsilon_{r}=6.5$ the propagation velocity, that is directly connected to the inter-peak distance in the frequency domain, (and is not included in Fig. 13) is not properly described in the time-domain representation, by some $4 \%$ percent. This discrepancy is presumably stemming from plate to plate variations or slight material anisotropies, and it has to be understood as the systematic uncertainty present in these set of measurements.

\section{Electrostatic compensation in N-strip structures}

A literal solution for N-strips, like the one given in [2] for 2-strips, could be useful for counter optimization. Besides, it is important to understand to which extent the ideas introduced in previous section for the simple 2-strip case can be applied to an N-strip situation. Inspired by the work of Romeo and Santomauro [42], we propose to approximate $\hat{C}, \hat{C}_{0}$ by tri-diagonal matrices. Translational invariance across the strips is also assumed, so that the electrostatic couplings of each strip are the same with respect to its neighbors and ground.

For simplicity we study the 5-strip case that, we believe, contains the essential features of an N-strip RPC system. Thus, our input matrices have the form:

$$
\hat{X}=\left(\begin{array}{ccccc}
X_{0} & -X_{m} & 0 & 0 & 0 \\
-X_{m} & X_{0} & -X_{m} & 0 & 0 \\
0 & -X_{m} & X_{0} & -X_{m} & 0 \\
0 & 0 & -X_{m} & X_{0} & -X_{m} \\
0 & 0 & 0 & -X_{m} & X_{0}
\end{array}\right)
$$

where $\hat{X}$ stands for either $\hat{C}$ or $\hat{C}_{0}$. In [42] indeed, the assumption was made that $\hat{L}$ itself was tri-diagonal instead of $\hat{C}_{0}$. It seems to us that the latter approach provides generally a higher accuracy for RPC structures and, besides, allows for slightly more compact analytical formulas; we will refer to this in the following as the 'short-range coupling' approximation. The structures of previous section, for instance, show a value for the coupling coefficient $C_{m} / C_{0} \sim$ $1 / 10$, while the (eventual) coupling to a second neighbor, in a translationally invariant structure, would be $C_{m 2} / C_{0} \lesssim$ $1 / 100$, so that assumption 45 is a priori justified. ${ }^{19} \mathrm{Be}-$ yond the 2-strip case, the 'short-range coupling' approximation implies an artificially low coupling to ground for the first and the last strips (at the sides of the structure). This is so because the $\hat{C}$-matrices are defined such that the capacitance to ground of strip $i$ satisfies the condition $C_{g, i}=\hat{C}_{i i}-\sum_{j=1, j \neq i}^{N} \hat{C}_{i j}, C_{i j}$ are defined negative, and those strips have one neighbor less. Signals produced at the boundary-strips of the structure will, therefore, show a deviation from the behavior predicted by this approximation. Their overall influence in propagation will be increasingly small as the number of strips increases. The exact solution to the diagonalization problem 13 under the 'short-range coupling' approximation (taking for commodity of operation the unitary representation) has a simple form independently from the particular values of the elements of the $\hat{C}$-matrices:

$\hat{M}=\hat{M}^{-1}=\frac{1}{2}\left(\begin{array}{ccccc}\frac{1}{\sqrt{3}} & -1 & \frac{2}{\sqrt{3}} & -1 & \frac{1}{\sqrt{3}} \\ -1 & 1 & 0 & -1 & 1 \\ \frac{2}{\sqrt{3}} & 0 & -\frac{2}{\sqrt{3}} & 0 & \frac{2}{\sqrt{3}} \\ -1 & -1 & 0 & 1 & 1 \\ \frac{1}{\sqrt{3}} & 1 & \frac{2}{\sqrt{3}} & 1 & \frac{1}{\sqrt{3}}\end{array}\right)$

and the components of $\vec{v}$ are:

$$
\begin{aligned}
\vec{v}= & c\left\{\sqrt{\frac{C_{00}+\sqrt{3} C_{m 0}}{C_{0}+\sqrt{3} C_{m}}}, \sqrt{\frac{C_{00}+C_{m 0}}{C_{0}+C_{m}}}, \sqrt{\frac{C_{00}}{C_{0}}},\right. \\
& \left.\sqrt{\frac{C_{00}-C_{m 0}}{C_{0}-C_{m}}}, \sqrt{\frac{C_{00}-\sqrt{3} C_{m 0}}{C_{0}-\sqrt{3} C_{m}}}\right\}
\end{aligned}
$$

\footnotetext{
${ }^{19}$ Note that $C_{0}=C_{g}+C_{m}$ in the 2-strip case. In general, it is not easy to strongly violate any of these two conditions for typical RPC designs. A remarkable exception is $[1]$, due the fact of $\mathrm{w} / h \ll 1$.
} 
The above velocity spectra becomes fully degenerated when the velocity dispersion, defined as eq. 16-right, equals zero and thus:

$\frac{C_{m}}{C_{0}}=\frac{C_{m 0}}{C_{00}}$

meaning that modal dispersion is exactly canceled, and all modes travel at a velocity given by $\bar{v}$ in eq. 16-left. Indeed, this crucial property can be easily generalized to an arbitrary number of strips. For this it is necessary to realize that the condition 48 implies, under the 'short-range coupling' approximation, that the matrices $\hat{C}$ and $\hat{C}_{0}$ differ in a multiplicative constant. Thus, the matrix to be diagonalized in the basic problem of eq. 13 becomes, after recalling eq. 15 , proportional to the unit-matrix, whose eigenvalues are obviously identical. This result means, precisely, that any inhomogeneous N-strip transmission line that is translationally invariant across the strips, does not consist of magnetic materials and falls under the short-range approximation will show no modal dispersion when it is electrostatically compensated. Interestingly, this will hold even if the coupling to the $1^{\text {st }}$ neighbor is high (low coupling approximation not fulfilled).

Despite the simplicity of the solution to the diagonalization problem in eqs. 46,47 , a tractable literal solution requires of further assumptions due to the need to obtain a simple expression for the transmission coefficient $\hat{T}$ in eq. 10. We propose the 'low coupling' assumption:

$\frac{C_{m}}{C_{0}}, \frac{C_{m 0}}{C_{00}}<1$

that is familiar from the 2-strip situation [2], where it has been defined through the equivalent condition $Z_{m} / Z_{c}<1$. It is also assumed 'moderate dispersion', that means that eq. 48 is always approximately satisfied. Under this latter assumption we keep the lowest order dispersive terms, that are the ones proportional to the difference of the traveling modes. At last, we inject a signal $I(t)$ at position $y_{0}$ along the second strip and obtain the transmitted and cross-talk signals in the $3^{\text {rd }}$ ( $1^{\text {st }}$ neighbor) and $4^{\text {th }}$ ( $2^{\text {nd }}$ neighbor) strips, in order to avoid side effects.

The literal solutions, omitting reflections, can be finally obtained:

$$
\begin{aligned}
I_{t r}(t) \simeq & \frac{T}{2} \frac{\Sigma_{1} I\left(t, y_{0}\right)+\Sigma_{2} I\left(t, y_{0}\right)}{2}+ \\
& \frac{Z_{m} R}{\left(Z_{c}+R\right)^{2}} \frac{\sqrt{3} \Delta_{1} I\left(t, y_{0}\right)+\Delta_{2} I\left(t, y_{0}\right)}{2} \\
I_{c t, 1}(t) \simeq & \frac{Z_{m} R}{\left(Z_{c}+R\right)^{2}} \Sigma_{1} I\left(t, y_{0}\right)+\frac{T}{2 \sqrt{3}} \Delta_{1} I\left(t, y_{0}\right) \\
I_{c t, 2}(t) \simeq & \frac{\left(Z_{m} R\right)^{2}}{\left(Z_{c}+R\right)^{3}} \frac{1}{Z_{c}} \frac{\Sigma_{1} I\left(t, y_{0}\right)+\Sigma_{2} I\left(t, y_{0}\right)}{2}+ \\
& \frac{T}{2} \frac{\Sigma_{1} I\left(t, y_{0}\right)-\Sigma_{2} I\left(t, y_{0}\right)}{2}
\end{aligned}
$$

with:

$$
\begin{aligned}
& \Sigma_{1(2)} I\left(t, y_{0}\right)=\frac{1}{2}\left[I\left(t-\frac{y_{0}}{v_{5(4)}}\right)+I\left(t-\frac{y_{0}}{v_{1(2)}}\right)\right] \\
& \Delta_{1(2)} I\left(t, y_{0}\right)=\frac{1}{2}\left[I\left(t-\frac{y_{0}}{v_{5(4)}}\right)-I\left(t-\frac{y_{0}}{v_{1(2)}}\right)\right]
\end{aligned}
$$

The structure of the solutions in eqs. 50-52 highlights the formal analogy with the literal solutions to the 2 -strip problem given in [2]. When the system is compensated and reflections are neglected, transmission becomes independent from the signal shape, the propagation distance and the position and thus:

$$
\begin{aligned}
& F_{t r}=\frac{T}{2} \\
& F_{c t, 1}=\left[\frac{R}{Z_{c}+R}\right] \frac{Z_{m}}{Z_{c}} \\
& F_{c t, 2}=\left(\left[\frac{R}{Z_{c}+R}\right] \frac{Z_{m}}{Z_{c}}\right)^{2}=F_{c t, 1}^{2}
\end{aligned}
$$

implying a flat time (and frequency) response.

In order to illustrate the power of the electrostatic compensation technique we have performed simulations for two realistic 5 -strip RPCs, each strip being $2.5 \mathrm{~cm}$-wide. One of the structures is a 2 -gap/3-glass micro-strip structure (like in previous sections), but the ground plane has been also segmented and consists of 5 strips identical to the signal strips. The second structure is a strip-line structure. Precisely, it is the same micro-strip structure but mirrored with respect to the signal plane. The inter-strip separation is as small as $1 \mathrm{~mm}$ in both cases. Compensation has been achieved in the first case by adding $0.35 \mathrm{~mm}$ thick glass over the signal plane $\left(\epsilon_{r}=5.5\right)$, while in the second case 2 additional layers of Teflon $\left(\epsilon_{r}=2.1\right), 0.48 \mathrm{~mm}$-thick, have been added up and down with respect to the signal plane (Fig. 14). A compilation of the main elements of the capacitance matrices is given in table 2 .

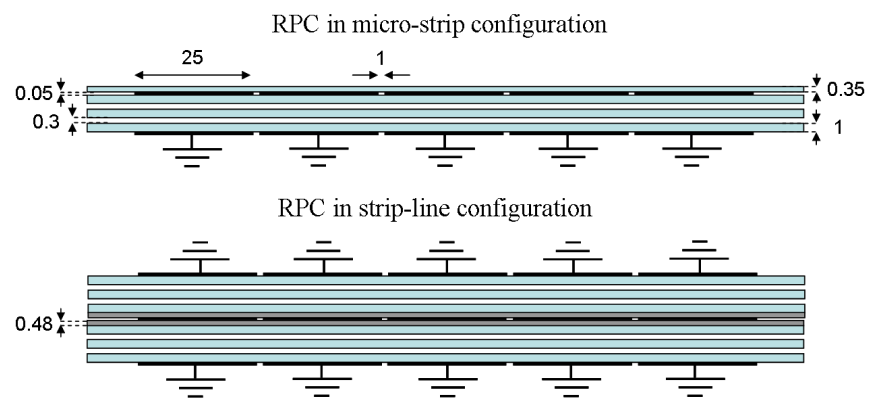

Fig. 14. Two electrostatically compensated 5-strip RPCs studied in this section.

Figs. 15 show the results of the proposed optimization on 5 -strip structures, serving also as a comparison between the exact solutions (left) and the literal solutions given in eqs. 50-52 (right). The same signal used in the measurements of previous section has been injected at the end of a $2 \mathrm{~m}$-long counter, and reflections have been omitted. Compensation has been 'optically' achieved in both micro-strip and strip-line configurations, although the cross-talk level 


\begin{tabular}{|c|c|c|c|c|c|c|}
\hline structure & $C_{0}$ & $C_{m}$ & $C_{m 2}$ & $C_{00}$ & $C_{m 0}$ & $C_{m 02}$ \\
\hline micro-strip (uncomp.) & 276.2 & 36.1 & 1.23 & 98.7 & 15.7 & 1.21 \\
\hline micro-strip (comp.) & 299.7 & 47.8 & 1.26 & 98.7 & 15.7 & 1.21 \\
\hline strip-line (uncomp.) & 499.8 & 49.6 & 0.0006 & 144.9 & 8.9 & 0.00035 \\
\hline strip-line (comp.) & 390.0 & 28.3 & 0.00036 & 131.4 & 9.5 & 0.00026 \\
\hline
\end{tabular}

Compilation of the main capacitive elements, in $\mathrm{pF} / \mathrm{m}$, for various 5-strip RPCs under the following definitions $C_{0} \equiv \hat{C}_{33}, C_{m} \equiv-\hat{C}_{23}$, $C_{m 2} \equiv-\hat{C}_{13}$, and similarly for the elements of matrix $\hat{C}_{0}$.
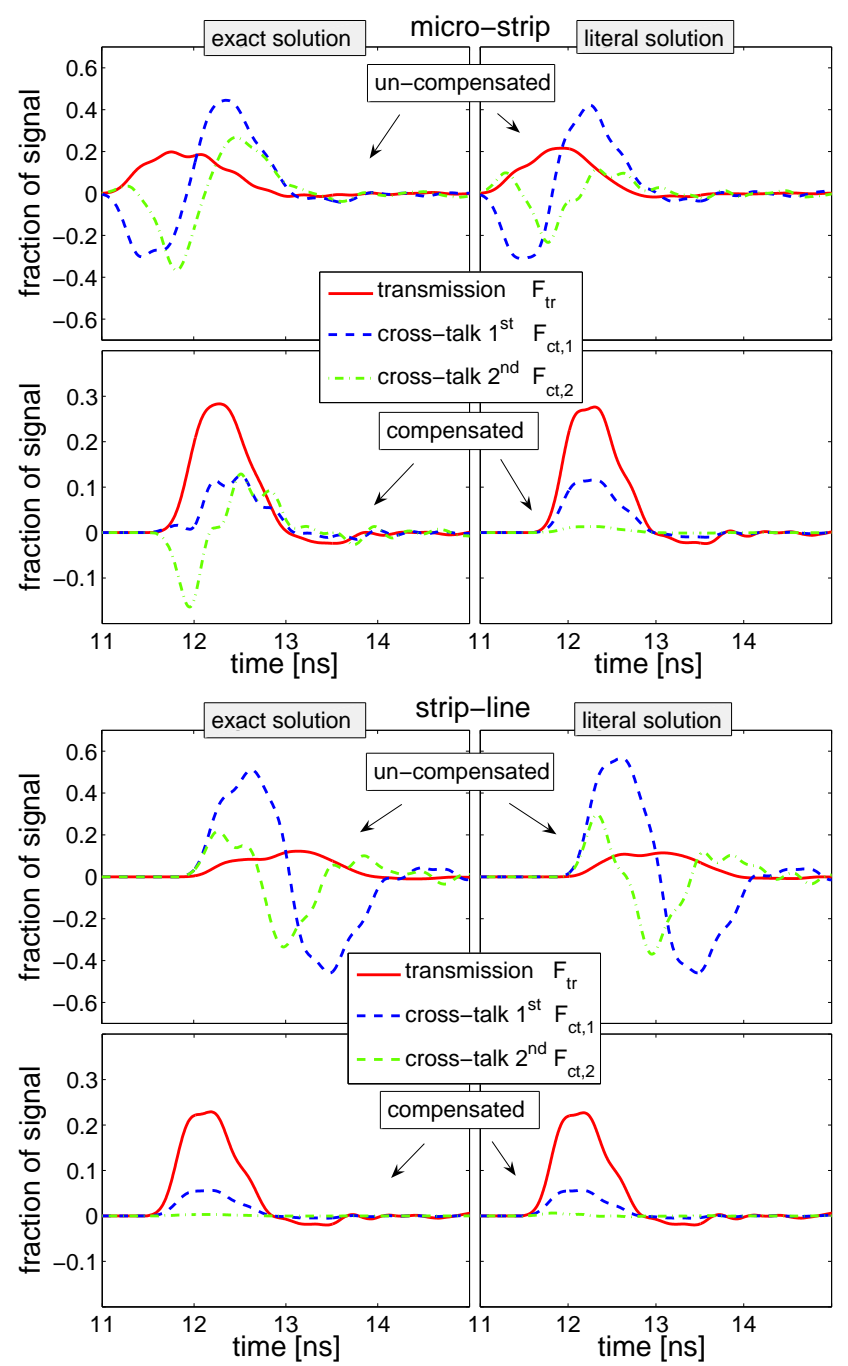

Fig. 15. Up-set: Simulations for transmission along 2m-long 5-strip RPC in a micro-strip configuration as described in text, with $2.5 \mathrm{~cm}$-wide strips placed as close as $1 \mathrm{~mm}$. The left column shows the exact solution and the right one the literal solutions given in text. The upper row stands for a non-compensated case and the lower one for a compensated one. The fraction of transmitted signal (red, continuous), fraction of cross-talk to the first neighbor (blue, dashed) and fraction of cross-talk to the second neighbor (green, dot-dashed) are shown. Low-set: like the upper set but for a 5-strip strip-line configuration as described in text, also with $2.5 \mathrm{~cm}$ width and $1 \mathrm{~mm}$ inter-strip separation.

stays higher in the micro-strip case than in the strip-line one. This is because of the presence of two close-by ground planes in the strip-line configuration, unlike the micro-strip, thus confining the mutual coupling field to a smaller volume in the former case and reducing the coupling coefficient (table 2). Besides, the coupling to the second neighbor is almost nonexistent in this strip-line structure, making the 'short-range coupling' approximation virtually exact, unlike the micro-strip. Because of that, compensation is exactly achieved, and the cross-talk to the second neighbor is as small as $F_{c t, 2}=F_{c t, 1}^{2} \simeq 0.05^{2}$, from eq. 57 . Due to the higher coupling to the second neighbor, this situation is not reached in the micro-strip configuration, showing $F_{c t, 2} \sim$ $F_{c t, 1}$.

\section{Discussion}

The measurements performed in sections 4 and 5 are equivalent within the limits of the precision of the corresponding apparatuses. This equivalence has been here presented by using the measured frequency response in Fig. 11 to simulate the time-domain measurements in Figs. 12, 13. Similarly, it is possible to use the Fourier-transformed timedomain measurements in section 4 to describe the measurements performed with the network analyzer in section 5 . This has been done and shows naturally a good agreement, but with a much lower precision, specially above $1.5 \mathrm{GHz}$, where the frequency components of the pulser used (280ps rise-time) are much suppressed, and sensitivity is lost. In particular, this approach does not allow to isolate neither the losses nor the deviations from a TEM description as precisely as it has been done with the network analyzer data. Besides, Fourier-Transformed time-domain data has a much lower frequency sampling as compared with the measurements from the network analyzer.

Despite we have found evidence of systematic variations of both the glass loss-tangent $\left.\tan \delta\right|_{\text {glass }}=0.25 \pm 0.05$ and its dielectric constant $\epsilon_{r}=6 \pm 0.5$, a very satisfactory description of several data taken in different conditions has been obtained. Additionally, 'electrostatic compensation' has been experimentally demonstrated for 1 and 2-gap structures allowing to obtain a factor $\times 12$ reduction in cross-talk for a typical signal as measured with a $\sim 2 \mathrm{GHz}$ bandwidth system. Thus, as it seems, the frequency-dependence of the parameters, eventual anisotropies, as well as plate to plate variations and/or line non-uniformities, in general, do not represent an obvious limitation when trying to compensate RPC structures. Moreover, an 'a priori' design seems possible, if tight mechanical tolerances at the $0.1 \mathrm{~mm}$ scale are observed.

Transmission along the compensated systems here developed was limited only by the dielectric losses. As shown, however, losses depend little on the system geometry for wide structures, so there is no much room for optimization. The most obvious (and maybe only) way consists on reducing the glass thickness or adding an additional material with a sizable series capacitance and low losstangent (Teflon, for instance), that imply a reduction of $\tan \delta^{*}$ through the factor $F$. If a proper selection of the 
glass is included, it seems likely that the bandwidth of a compensated RPC based on float glass could be extended virtually to the limit imposed by the intrinsic signal risetimes $(3 \mathrm{GHz})$ up to $2 \mathrm{~m}$.

It must be noted that this is the first experimental verification of the existence of modal dispersion in RPC structures, besides the indirect results in [2]. According to our simulations, the band-stop region stemming from modal dispersion is centered around $600 \mathrm{MHz}$ for the structures previously studied in [21], far from the limit of the device used for those measurements $(200 \mathrm{MHz})$.

There are classical ideas on cross-talk minimization that have been also studied prior to the elaboration of this work and will be here described generally. A final quantitative evaluation is much system-dependent so only general arguments are given:

(i) A ground plane or a cage close to the strip can arbitrarily reduce the coupling coefficient $Z_{m} / Z_{c}$, thus will always reduce cross-talk for short strips, at the expense of a reduced impedance and higher noise. However, compensation is not guaranteed by this procedure, so low cross-talk and high transmission can not be ensured for long counters by just observing this principle.

(ii) Shielding vias or the usage of additional materials with low dielectric constants can reduce the coupling coefficient $Z_{m} / Z_{c}$. They take, however, a very small portion of the available field lines, that propagate through the glass/gap structure anyhow. Modest improvements in transmission and cross-talk can be nevertheless achieved.

(iii) We have presented here single-ended signal measurements on single-ended architectures. On the other hand, differential signaling is known to give, in general, lower levels of cross-talk as compared to singleended signaling, and a faster decrease with the intertrace separation [43]. This is natural for large distances between each differential pair, since the coupling becomes then identical for both traces of the same pair. This is not, however, a typical situation for RPC structures and we have found so far no evidence that this principle prevails. It must be noted, however, that the signal induction process is indeed genuinely quasi-differential (signals with opposite polarities and similar amplitudes propagate simultaneously in anode and cathode of each strip). A comparison between differential signal injection (with ground strips let floating and terminated by ideal ground) and single-ended injection (where ground strips are assumed to be ideal ground) was performed in [2], showing a negligible difference. These subjects clearly deserve, nevertheless, further investigations.

\section{Conclusions}

We say that a strip counter is 'electrostatically compensated' when the coupling coefficient $Z_{m} / Z_{c} \simeq C_{m} / C_{0}$ is the same in the filled and in the empty structure. Equivalently, the capacitive and inductive coupling are balanced: $C_{m} / C_{0}=L_{m} / L_{0}$ and all the system velocities are equal. Propagation along a compensated structure shows minimal cross-talk and dispersion patterns and it is limited only by losses. This prediction has been experimentally demonstrated for $2 \mathrm{~m}$-long 2-strip RPCs, allowing for a factor $\times 12$ cross-talk reduction at $\simeq 2 \mathrm{GHz}$ bandwidth. Losses have been experimentally isolated for the first time on glass RPCs, resulting in a typical cutoff frequency of $f_{c}=$ $0.85 \mathrm{GHz}$ over $2 \mathrm{~m}$ and a loss-tangent $\tan \delta=0.025 \pm 0.005$ for float glass. A prescription on how to theoretically include losses has been given and compared with data, showing good agreement.

It has been shown that a condition sufficient for compensation to apply to any N-strip structure is that the coupling is 'short-range', meaning that the coupling to the second neighbor can be neglected. Several realistic configurations, most prominently a wide strip-line, satisfy this condition to a very large extent.

We expect that the literal solutions derived for the transmission and cross-talk to first and second neighbors can be useful for fast (albeit approximate) estimates of multi-strip transmission properties.

We have already started a systematic program aimed at implementing these ideas in large-area multi-strip counters.

\section{Acknowledgements}

DGD is supported by HIC for FAIR and NSFC (project 11050110111/A050506), CH was supported by BMBF and NSFC (project 11050110111/A050506). The authors want to acknowledge the GSI staff H. Flemming, H. Deppe, P. Moritz, M. Ciobanu, M. Freimuth and P. Kowina for discussions on this fascinating field and providing the necessary equipment, and to J. Hehner for his always competent technical help. Professional assistance with the matrix formalism from T. Rodríguez Frutos and K. Vartortout (also GSI) is acknowledged. N. Herrmann deserves credit for being the first to spot the improbable possibility of compensating modal dispersion.

\section{Appendix A. Charge conservation of the solutions to the loss-less 2-strip problem}

The general solution to the 2-strip loss-less problem for a signal injected in strip 1 when termination is performed with individual resistors of equal value is: 
$\vec{I}_{T}(t)=\frac{\hat{T}}{2} \sum_{j=0}^{\infty}(1-\hat{T})^{j} \hat{M}\left(\begin{array}{l}\hat{M}_{11}^{-1} I\left(t-\frac{(-1)^{j} y_{0}+2\lceil j / 2\rceil D}{v_{1}}\right) \\ \hat{M}_{12}^{-1} I\left(t-\frac{(-1)^{j} y_{0}+2\lceil j / 2\rceil D}{v_{2}}\right)\end{array}\right)$

that was given in [2]. ${ }^{20}$ The charge finally transmitted through each line for a signal injected in strip 1 can be obtained after integration of eqs. A.1 over infinite time, yielding:

$\vec{q}_{T}=\frac{\hat{T}}{2} \sum_{j=0}^{\infty}(1-\hat{T})^{j} \hat{M}\left(\begin{array}{c}\hat{M}_{11}^{-1} q \\ \hat{M}_{12}^{-1} q\end{array}\right)$

where $q$ is the charge conveyed by the induced signal $I(t)$. For simplicity we take the unitary representation:

$\hat{M}=\hat{M}^{-1}=\frac{1}{\sqrt{2}}\left(\begin{array}{cc}1 & 1 \\ 1 & -1\end{array}\right)$

and define the reflection coefficient $\hat{\Gamma}=1-\hat{T}$. The infinite sum in eq. A.2 can be performed by working in the diagonal representation (denoted by '):

$\vec{q}_{T}=\frac{1}{2} \hat{M} \hat{T}^{\prime} \hat{M}^{-1} \sum_{j=0}^{\infty} \hat{M} \hat{\Gamma}^{\prime j} \hat{M}^{-1} \hat{M}\left(\begin{array}{c}\hat{M}_{11}^{-1} q \\ \hat{M}_{12}^{-1} q\end{array}\right)$

It is easy to show that:

$\hat{\Gamma}^{\prime}=\left(\begin{array}{cc}\Gamma_{11}-\Gamma_{12} & 0 \\ 0 & \Gamma_{11}+\Gamma_{12}\end{array}\right) ; \quad \hat{T}^{\prime}=1-\hat{\Gamma}^{\prime}$

Since $\hat{\Gamma}^{\prime}$ is a diagonal matrix, it is very simple to take powers of it. Expression A.4 can be thus written in a convenient form as:

$$
\begin{aligned}
\vec{q}_{T}= & \frac{1}{2} \hat{M} \hat{T}^{\prime} \hat{M}^{-1} \hat{M}\left(\begin{array}{cc}
\sum_{j=0}^{\infty}\left(\Gamma_{11}-\Gamma_{12}\right)^{j} & 0 \\
0 & \sum_{j=0}^{\infty}\left(\Gamma_{11}+\Gamma_{12}\right)^{j}
\end{array}\right) \\
& \times \hat{M}^{-1} \hat{M}\left(\begin{array}{c}
\hat{M}_{11}^{-1} q \\
\hat{M}_{12}^{-1} q
\end{array}\right)
\end{aligned}
$$

After performing the geometric sum and grouping terms:

$\vec{q}_{T}=\frac{1}{2} \hat{M}\left[\hat{T}^{\prime}\left(\begin{array}{cc}\frac{1}{1-\left(\Gamma_{11}-\Gamma_{12}\right)} & 0 \\ 0 & \frac{1}{1-\left(\Gamma_{11}+\Gamma_{12}\right)}\end{array}\right)\right]\left(\begin{array}{l}\hat{M}_{11}^{-1} q \\ \hat{M}_{12}^{-1} q\end{array}\right)$

where the term in brackets is the identity matrix. Thus:

$\vec{q}_{T}=\frac{1}{2} \hat{M}\left(\begin{array}{c}\hat{M}_{11}^{-1} \\ \hat{M}_{12}^{-1}\end{array}\right) q$

\footnotetext{
$\overline{20}$ There is indeed an erratum in formula (12) in ref [2], the matrix
} $\hat{T}$ should appear before the sum, as in eq. A.1. that finally yields:

$$
\vec{q}_{T}=\frac{1}{2}\left(\begin{array}{l}
1 \\
0
\end{array}\right) q
$$

as we wanted to demonstrate. The driven line carries away $1 / 2$ of the signal per each strip end, while the un-driven line does not carry net charge. The result requires of all reflections to be considered.

\section{Appendix B. Current vs voltage generators in TLs}

Perhaps the clearest explanation of the relation between pulser measurements and ideal current injection can be obtained through Fig. B.1 for a 1-strip case. For the sake of clarity, we assume that the line is loss-less in the following reasoning.

a)

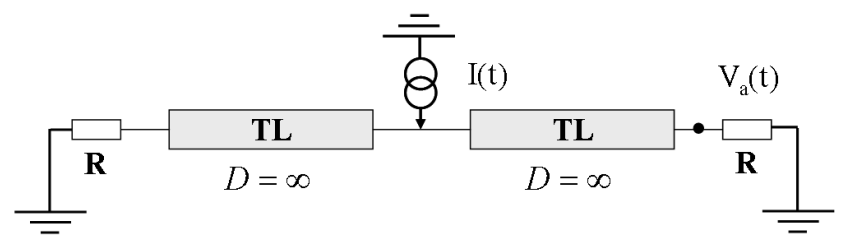

b)

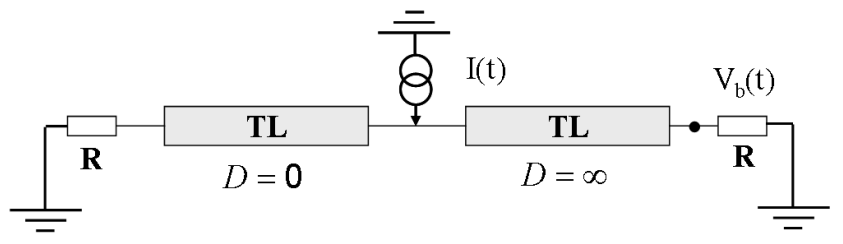

c)

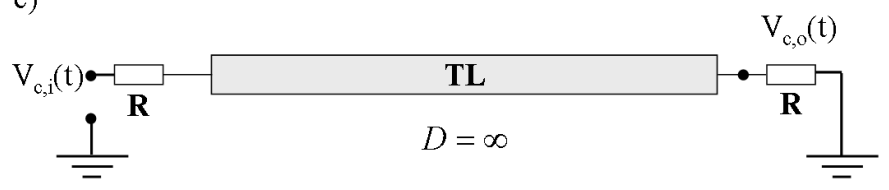

Fig. B.1. The three cases discussed for illustrating the equivalence between current and voltage sources.

Solutions to cases a) and b) can be directly obtained, without solving eq. 10 . by properly combining the transmission and reflection coefficients:

$$
\begin{aligned}
V_{a}(t) & =\frac{I(t)}{2} \times T_{i-o} \times R \\
V_{b}(t) & =\frac{I(t)}{2} \times\left(1+\left(1-T_{i-o}\right)\right) \times T_{i-o} \times R \\
& =\frac{I(t)}{2} \times T_{o-i} \times T_{i-o} \times R
\end{aligned}
$$

where:

$$
\begin{aligned}
T_{i-o} & =\frac{2 Z_{c}}{Z_{c}+R} \\
T_{o-i} & =\frac{2 R}{Z_{c}+R}
\end{aligned}
$$


are the transmission coefficients from in-out and out-in of the transmission line (TL), respectively. All terminating resistors are equal. Under the typical situation $Z_{c}<R$, it is verified that $T_{i-o}<1$ and $T_{o-i}>1$. According to the definitions in text:

$$
\begin{aligned}
& F_{t r, a}(t)=\frac{1}{2} \frac{I(t)}{\max [I(t)]} \times T_{i-o} \times R \\
& F_{t r, b}(t)=\frac{1}{2} \frac{I(t)}{\max [I(t)]} \times T_{o-i} \times T_{i-o} \times R
\end{aligned}
$$

Perhaps counter-intuitively, transmission at the center of an un-matched loss-less strip (with $Z_{c}<R$ ) will be always smaller than at its ends ends, when looking at the signal collected at the opposite end. The factor between the two situations is $T_{o-i}$ and arises from the constructive interference between the direct and reflected waves.

The solution in situation c) is also easy to find, being:

$V_{c, o}(t)=\frac{V_{c, i}(t)}{2 R} \times T_{o-i} \times T_{i-o} \times R$

The voltage measured in absence of TL or with it being adapted is thus:

$V(t)=V_{c, i}(t) / 2$

as for a perfect voltage divider. The correspondence between situations b) and c) (eq. B.2 and eq. B.7) can be obtained through the equivalence:

$I(t) \equiv \frac{V_{c, i}(t)}{R}$

or:

$I(t) \equiv 2 \frac{V(t)}{R}$

that is eq. 21 in text. It gives the equivalence between the current induced at one end of a TL, $I(t)$, and a pulser measurement, being $V(t)$ the voltage drop measured in a matched load. Thus, the solutions for the former case in eq. 10 can be directly applied for a pulser measurement after making the equivalence B.10.

\section{References}

[1] A. Schuettauf, Nucl. Instr. Meth. A, 602(2009)679.

[2] D. Gonzalez-Diaz, doi:10.1016/j.nima.2010.09.067.

[3] C. R. Paul, 'Analysis of Multiconductor Transmission Lines', John Wiley \& Sons, 2008.

[4] J. de Urquijo et al., Eur. Phys. J. D, 51(2009)241.

[5] P. Camarri et al., Nucl. Instr. Meth. A, 414(1998)317.

[6] P. Fonte et al., Nucl. Instr. Meth. A, 48, 4(2000)201.

[7] M. Ciobanu et al., IEEE Trans. Nucl. Sci. 54, 4(2007)1201.

[8] D. Belver et al., IEEE Trans. Nucl. Sci. 57, 5(2010)2848.

[9] A. Blanco et al., IEEE Trans. Nucl. Sci. 48, 4(2001)1249.

[10] C. Lippmann and W. Riegler, Nucl. Instr. Meth. A, 517(2004)54.

[11] A. Blanco et al., Nucl. Instr. Meth. A, 485(2002)328.

[12] M. Abbrescia et al. Nucl. Instr. Meth. A, 593(2008)263.

[13] I. Deppner et al., doi:10.1016/j.nima.2010.09.165.

[14] C. Caesar et al., doi:10.1016/j.nima.2010.09.163.
[15] E. Casarejos et al., doi:10.1016/j.nima.2010.09.069.

[16] W. Llope, doi:10.1016/j.nima.2010.07.086.

[17] A. Blanco et al., doi:10.1016/j.nima.2010.08.068.

[18] M. Kis, 'FOPI MMRPC ToF Barrel', talk given at the X RPC Workshop, GSI-Darmstadt.

[19] P. Steinhaeuser et al., Nucl. Instr. Meth. A, 390(1997)86.

[20] V. Ammosov et al., Nucl. Instr. Meth. A, 456(2000).

[21] W. Riegler et al., Nucl. Instr. Meth. A, 481(2002)130.

[22] T. Heubrandtner et al., Nucl. Instr. Meth. A, 489(2002)443.

[23] W. Riegler, Nucl. Instr. Meth. A, 491(2002)258.

[24] W. Riegler, Nucl. Instr. Meth. A, 535(2004)287.

[25] A. Akindinov et al., Il nuovo Cimento B, 124-02(2009)235.

[26] S. Ramo, PROC. IRE 27(1939)584.

[27] C. Wei et al., Trans. Micr. Theor. and Tech. 32(1984) 439.

[28] H. Alvarez-Pol et al., Nucl. Instr. Meth. A, 535(2004)277.

[29] H. Alvarez-Pol et al., Nucl. Instr. Meth. A, 533(2004)79.

[30] M. Ciobanu et al., doi:10.1109/NSSMIC.2009.5401675.

[31] P. Senger et al., J. Phys. G, 36(2009)064037.

[32] http://www.gsi.de/forschung/kp/kr/R3B/ Technical\%20Proposal.pdf. Accessed on 07-07-2010.

[33] http://web.awrcorp.com/Usa/Products/APLAC. Accessed on 11-11-2010.

[34] G. I. Zysman, A. Kent Johnson, IEEE Trans. Micr. Theo. Techn. 17-10(1969)753.

[35] A. Feller, H. R. Kaupp, J. J. Digiacomo, 'Cross-talk and reflections in high-speed digital systems', proc. to the Fall Joint Computer Conference, 1965.

[36] http://www.wolfram.com/products/mathematica/index.html. Accessed on 11-11-2010.

[37] C. R. Paul, IEEE Trans. Elec. Comp. 44-3(2002)413.

[38] http://www.ansoft.com/news/press_release/051129.cfm. Accessed on 11-11-2010.

[39] V. D. Frechette, 'Non Crystalline Solids', Wiley, New York (1960).

[40] R. E. Collins, 'Foundations for Microwave engeneering', John Wiley \& Sons, 2000.

[41] A. R. Djordjevic, T. K. Sarkar, IEEE Trans. Micr. Theo. Techn. 35-10(1987)898.

[42] F. Romeo and M. Santomauro, IEEE Trans. Micr. Theo. Techn. 35-2(1987)132.

[43] F. Xiao et al., IEICE Trans. Commun., E90-B, 6(2007)1305. 